\title{
Article \\ Pharmacological Properties, Volatile Organic Compounds, and Genome Sequences of Bacterial Endophytes from the Mangrove Plant Rhizophora apiculata Blume
}

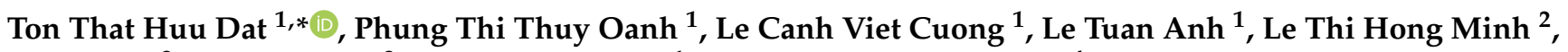 \\ Hoang $\mathrm{Ha}^{3}$, Le Tung Lam ${ }^{3}$, Pham Viet Cuong ${ }^{1, *}$ and Hoang Le Tuan Anh ${ }^{4, *}$ \\ 1 Mientrung Institute for Scientific Research, Vietnam National Museum of Nature, Vietnam Academy of \\ Science and Technology, 321 Huynh Thuc Khang, Hue City 49117, Vietnam; \\ oanh5794@vnmn.vast.vn (P.T.T.O.); lcvcuong@vnmn.vast.vn (L.C.V.C.); ltanh@vnmn.vast.vn (L.T.A.) \\ 2 Institute of Marine Biochemistry, Vietnam Academy of Science and Technology, 18 Hoang Quoc Viet, \\ Cau Giay, Hanoi 10072, Vietnam; lhminh@imbc.vast.vn \\ 3 Institute of Biotechnology, Vietnam Academy of Science and Technology, 18 Hoang Quoc Viet, Cau Giay, \\ Hanoi 10072, Vietnam; hoanghapcb@ibt.ac.vn (H.H.); letunglam@ibt.ac.vn (L.T.L.) \\ 4 Center for Research and Technology Transfer, Vietnam Academy of Science and Technology, \\ 18 Hoang Quoc Viet, Cau Giay, Hanoi 10072, Vietnam \\ * Correspondence: tthdat@vnmn.vast.vn (T.T.H.D.); phamvc1965@vnmn.vast.vn (P.V.C.); \\ hltanh@ctctt.vast.vn (H.L.T.A.);

Citation: Dat, T.T.H.; Oanh, P.T.T.; Cuong, L.C.V.; Anh, L.T.; Minh, L.T.H.; Ha, H.; Lam, L.T.; Cuong, P.V.; Anh, H.L.T. Pharmacological Properties, Volatile Organic Compounds, and Genome Sequences of Bacterial Endophytes from the Mangrove Plant Rhizophora apiculata Blume. Antibiotics 2021, 10, 1491. https://doi.org/10.3390/ antibiotics10121491

Academic Editor: Manuel Simões

Received: 14 November 2021 Accepted: 3 December 2021 Published: 5 December 2021

Publisher's Note: MDPI stays neutral with regard to jurisdictional claims in published maps and institutional affiliations.

Copyright: () 2021 by the authors Licensee MDPI, Basel, Switzerland. This article is an open access article distributed under the terms and conditions of the Creative Commons Attribution (CC BY) license (https:// creativecommons.org/licenses/by/ $4.0 /)$.
Abstract: Mangrove plant endophytic bacteria are prolific sources of bioactive secondary metabolites. In the present study, twenty-three endophytic bacteria were isolated from the fresh roots of the mangrove plant Rhizophora apiculata. The identification of isolates by $16 \mathrm{~S}$ rRNA gene sequences revealed that the isolated endophytic bacteria belonged to nine genera, including Streptomyces, Bacillus, Pseudovibrio, Microbacterium, Brevibacterium, Microbulbifer, Micrococcus, Rossellomorea, and Paracoccus. The ethyl acetate extracts of the endophytic bacteria's pharmacological properties were evaluated in vitro, including antimicrobial, antioxidant, $\alpha-$ amylase and $\alpha$-glucosidase inhibitory, xanthine oxidase inhibitory, and cytotoxic activities. Gas chromatography-mass spectrometry (GC-MS) analyses of three high bioactive strains Bacillus sp. RAR_GA_16, Rossellomorea vietnamensis RAR_WA_32, and Bacillus sp. RAR_M1_44 identified major volatile organic compounds (VOCs) in their ethyl acetate extracts. Genome analyses identified biosynthesis gene clusters (BGCs) of secondary metabolites of the bacterial endophytes. The obtained results reveal that the endophytic bacteria from $R$. apiculata may be a potential source of pharmacological secondary metabolites, and further investigations of the high bioactive strains—such as fermentation and isolation of pure bioactive compounds, and heterologous expression of novel BGCs in appropriate expression hosts-may allow exploring and exploiting the promising bioactive compounds for future drug development.

Keywords: biological activity; biosynthesis gene clusters; endophytic bacteria; GC-MS; genome; Rhizophora apiculata

\section{Introduction}

Plant endophytic bacteria are live and thrive inside plants without causing harmful effects to their host plants [1]. The endophytic bacteria provide numerous benefits to their host plants, including promoting the growth of host plants, enhancing the resistance of host plants against diseases, and increasing the tolerance of host plants to stressful environmental conditions [2-4]. Endophytic bacteria have been found in different parts of plants, such as roots, stems, leaves, seeds, fruits, tubers, ovules and nodules; however, 
roots contain the highest number of bacterial endophytes when compared to other plant parts $[4,5]$.

The mangrove ecosystem is characterized by highly changeable environmental conditions such as salinity, temperature, nutrients, and tidal currents, making it one of the most productive ecosystems [6]. Mangrove plants growing under such frequent and sporadic environmental changes provide special ecological niches for diverse endophytic microbial communities [7]. In addition, the variable environmental conditions in mangrove ecosystems may serve as excellent selectors for structurally distinct secondary metabolites with intriguing pharmacological effects. Along with their host plants, endophytic bacteria have also been reported to be a rich source of secondary metabolites with promising pharmacological properties [8-11]. Of these, many compounds exhibit potent biological activities and may be considered as lead compounds for further drug developments.

Rhizophora apiculata is a "true" mangrove plant belonging to the genus Rhizophora, of the family Rhizophoraceae. The genus Rhizophora is generally found in intertidal zones and is distributed in both the Indo-West Pacific and Atlantic East Pacific regions [12]. Phytochemical investigations on $R$. apiculata reveal its structurally diverse secondary metabolites, including alkaloids, terpenoids, flavonoids, aliphatic alcohols, and phenolic derivatives [13-17]. The isolated compounds from this species have been reported to exhibit promising pharmacological properties, such as antimicrobial, antiviral, antioxidant, antidiabetic, anticancer, and hepatoprotective activities [15,18-23]. Apart from R. apiculata, its endophytic microorganisms produce various secondary metabolites with valuable biological properties such as antimicrobial, antiviral, and anticancer activities [11,24-26].

With long coastlines up to $3260 \mathrm{~km}$, coastal mangrove forests are among the most important ecosystems in Vietnam. The Vietnamese mangrove forests cover an area of 155,290 ha with 109 mangrove plant species. "True" mangrove plants consist of 37 species belonging to 20 genera of 14 families, whereas "associate" mangrove plants consist of 72 species belonging to 36 genera of 28 families. Among them, the most dominant mangrove plants are species of the genus Rhizophora [27]. In the present study, we isolated endophytic bacteria from the fresh roots of R. apiculata collected in the mangrove forest of $\mathrm{Bu} \mathrm{Lu}$, Phu Loc district, Thua Thien Hue province, Vietnam (Figure 1), and evaluated their potential pharmacological properties. The composition of volatile organic compounds present in the high bioactive extracts was determined by gas chromatography-mass spectrometry (GC-MS) analyses. Additionally, the biosynthesis gene clusters of secondary metabolites were discovered by the genome analysis. The obtained results in the present study reveal that the endophytic bacteria from Rhizophora apiculata are potential sources of biological compounds and further investigations, e.g., isolation of active pure compounds and heterologous expression of novel biosynthesis gene clusters of secondary metabolites should be performed in order to explore and exploit the novel bioactive compounds for drug development. 

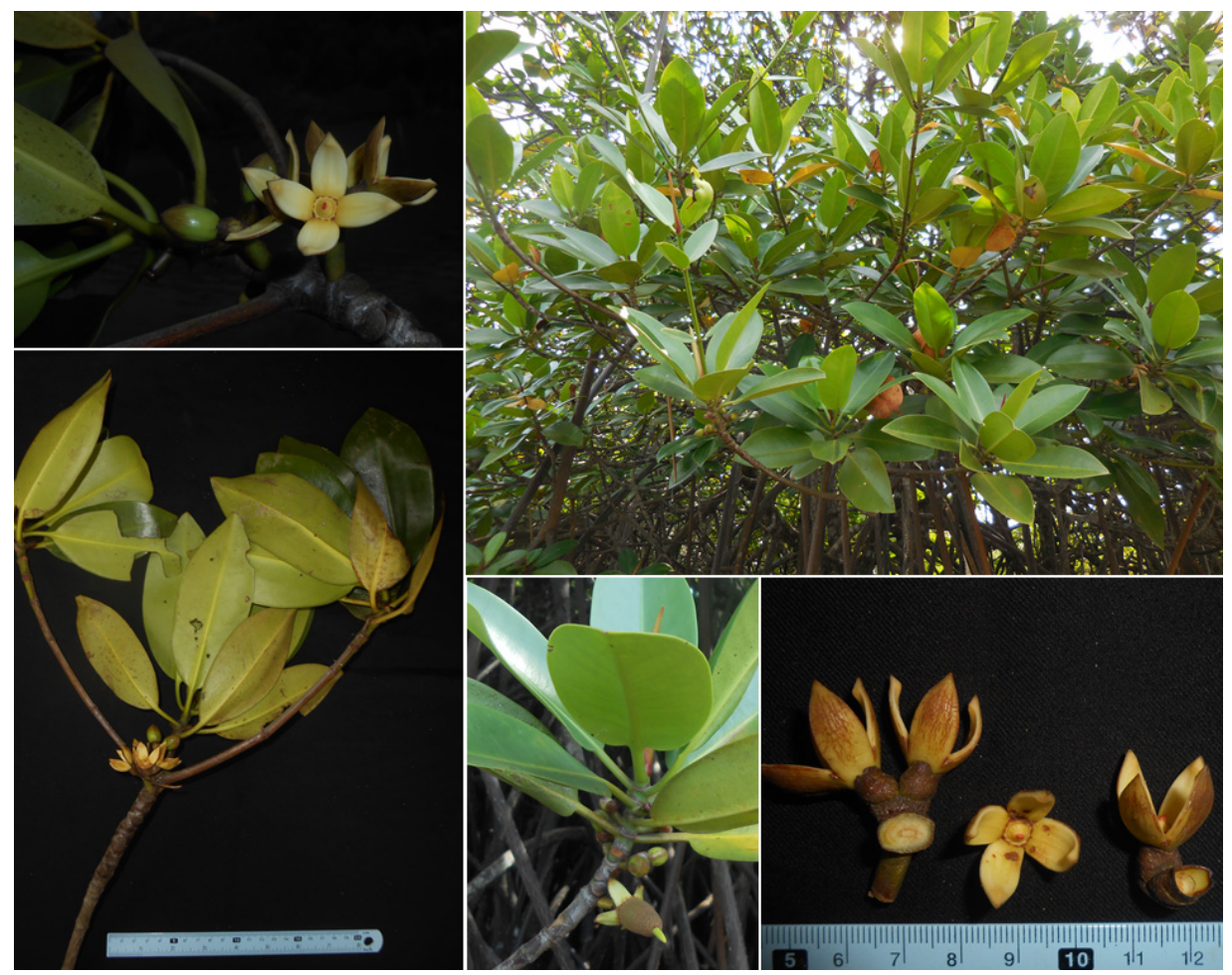

Figure 1. Rhizophora apiculata collected from the Bu Lu mangrove forest, Phu Loc district, Thua Thien Hue province, Vietnam.

\section{Results}

\subsection{Isolation and Identification of Endophytic Bacteria}

Twenty-three endophytic bacterial strains were isolated from the fresh roots of the mangrove plant Rhizophora apiculata. Based on their 16S rRNA gene sequences, the bacterial strains were identified as belonging to nine different genera, i.e., Streptomyces, Bacillus, Pseudovibrio, Microbacterium, Rossellomorea, Brevibacterium, Microbulbifer, Micrococcus, and Paracoccus (Table 1). The phylogenic tree based on their 16S rRNA gene sequences is shown in Figure 2. All isolated strains were cultured in nutrient broth and their ethyl acetate extracts were prepared for evaluating their potential pharmacological properties.

Table 1. Identification of the endophytic bacterial strains by $16 \mathrm{~S}$ rRNA gene sequences.

\begin{tabular}{|c|c|c|c|}
\hline Strain ID & $\begin{array}{c}\text { Identification by 16S rRNA } \\
\text { Gene Sequence }\end{array}$ & $\begin{array}{l}\text { NCBI BLAST } \\
\text { Identity }(\%)\end{array}$ & Accession Number \\
\hline RAR_GA_12 & Streptomyces sp. & 99.8 & MT229086 \\
\hline RAR_GA_16 & Bacillus sp. & 100 & OK649239 \\
\hline RAR_WA_18 & Bacillus sp. & 99.8 & MT229092 \\
\hline RAR_M1_23 & Pseudovibrio sp. & 99.7 & MT229094 \\
\hline RAR_M1_26 & Bacillus sp. & 99.6 & MT229093 \\
\hline RAR_GA_31 & Microbacterium sp. & 99.9 & MT229099 \\
\hline RAR_WA_32 & Rossellomorea vietnamensis & 100 & OK649240 \\
\hline RAR_M1_36 & Brevibacterium sp. & 99.9 & MT229096 \\
\hline RAR_M1_41 & Microbulbifer sp. & 99.8 & MT229085 \\
\hline RAR_GA_42 & Micrococcus sp. & 99.9 & MT229088 \\
\hline RAR_M1_44 & Bacillus sp. & 100 & OK649238 \\
\hline RAR_M1_45 & Bacillus sp. & 99.9 & MT229100 \\
\hline RAR_M1_49 & Paracoccus sp. & 99.9 & MT229090 \\
\hline RAR_WA_50 & Bacillus sp. & 99.8 & MT229104 \\
\hline RAR_M1_53 & Streptomyces sp. & 99.9 & MT229089 \\
\hline RAR_M1_54 & Streptomyces sp. & 100 & MT229103 \\
\hline
\end{tabular}


Table 1. Cont.

\begin{tabular}{lccc}
\hline Strain ID & $\begin{array}{c}\text { Identification by 16S rRNA } \\
\text { Gene Sequence }\end{array}$ & $\begin{array}{c}\text { NCBI BLAST } \\
\text { Identity (\%) }\end{array}$ & Accession Number \\
\hline RAR_GA_57 & Streptomyces sp. & 99.9 & MT229098 \\
RAR_M1_58 & Bacillus velezensis & 100 & MT229097 \\
RAR_M1_60 & Bacillus sp. & 99.9 & MT229102 \\
RAR_M1_61 & Bacillus velezensis & 100 & MT229087 \\
RAR_GA_63 & Streptomyces sp. & 99.7 & MT229095 \\
RAR_GA_64 & Streptomyces sp. & 100 & MT229101 \\
RAR_M1_66 & Microbacterium sp. & 99.8 & MT229091 \\
\hline
\end{tabular}

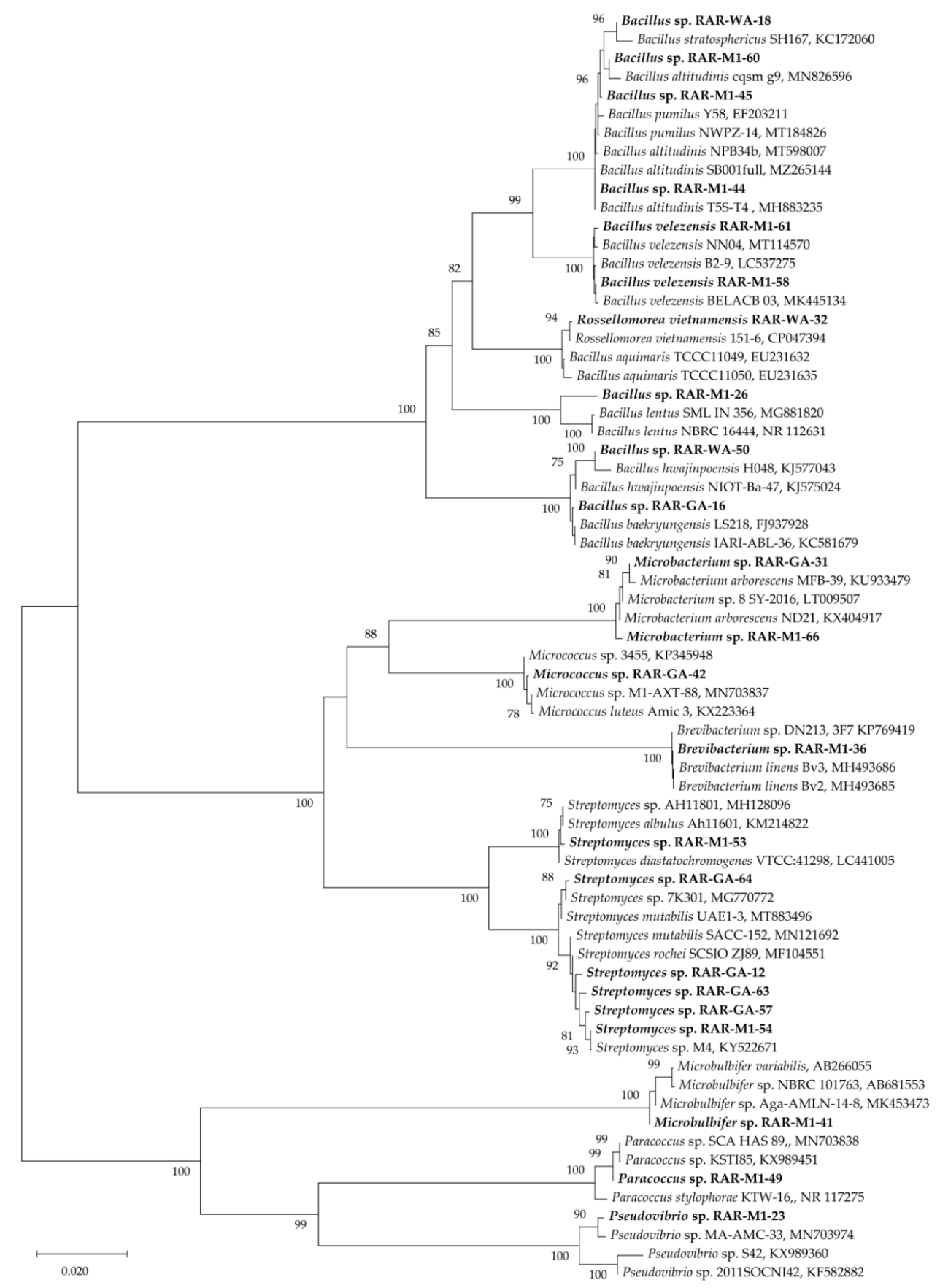

Figure 2. Phylogeny of $16 \mathrm{~S}$ rRNA gene sequences of the endophytic bacteria in our study (bold letters) and from the National Center for Biotechnology Information (NCBI) GenBank. Bootstrap support values of branches greater than $75 \%$ are given above the corresponding branches. 


\subsection{Pharmacological Evaluation of the Ethyl Acetate Extracts}

\subsubsection{Antimicrobial Activity}

The antimicrobial assays showed that 18 of 23 bacterial extracts exhibited antimicrobial activity against at least one of the tested microbes with minimum inhibitory concentration (MIC) values from 16-256 $\mu \mathrm{g} / \mathrm{mL}$ (Table 2). Among them, nine extracts exhibited antimicrobial activity against $S$. aureus, eleven extracts exhibited antimicrobial activity against $E$. faecalis, eight extracts exhibited antimicrobial activity against $E$. coli, eight extracts exhibited antimicrobial activity against $P$. aegurinosa, and ten extracts exhibited antimicrobial activity against $C$. albicans. Several extracts showed antimicrobial activity against multiple tested microbes, for example, three extracts (RAR_GA_16, RAR_WA_18, RAR_M1_44) exhibited antimicrobial activity against four tested microbes, eight extracts (RAR_GA_12, RAR_WA_32, RAR_M1_36, RAR_M1_45, RAR_WA_50, RAR_GA_57, RAR_M1_60, RAR_M1_66) exhibited antimicrobial activity against three tested microbes, three extracts (RAR_GA_31, RAR_M1_53, RAR_GA_63) exhibited antimicrobial activity against two tested microbes, and four extracts (RAR_M1_23, RAR_M1_54, RAR_M1_58, RAR_GA_64) exhibited antimicrobial activity against one tested microbe. Notably, several extracts showed significant antimicrobial activity with MIC values between 16 and $64 \mu \mathrm{g} / \mathrm{mL}$.

Table 2. Antimicrobial activity of the bacterial extracts (MIC, $\mu \mathrm{g} / \mathrm{mL}$ ).

\begin{tabular}{|c|c|c|c|c|c|}
\hline Strain ID & S. aureus & E. faecalis & E. coli & P. aegurinosa & C. albicans \\
\hline RAR_GA_12 & 64 & 128 & - & - & 64 \\
\hline RAR_GA_16 & 16 & 32 & 256 & - & 32 \\
\hline RAR_WA_18 & - & 64 & 128 & 256 & 256 \\
\hline RAR_M1_23 & 128 & - & - & - & - \\
\hline RAR_M1_26 & - & - & - & - & - \\
\hline RAR_GA_31 & - & - & - & 64 & 128 \\
\hline RAR_WA_32 & - & - & 32 & 64 & 128 \\
\hline RAR_M1_36 & 64 & 32 & 128 & - & - \\
\hline RAR_M1_41 & - & - & - & - & \\
\hline RAR_GA_42 & - & - & - & - & - \\
\hline RAR_M1_44 & 32 & 64 & 128 & - & 64 \\
\hline RAR_M1_45 & - & 128 & 128 & 256 & - \\
\hline RAR_M1_49 & - & - & - & - & - \\
\hline RAR_WA_50 & 32 & - & - & 64 & 64 \\
\hline RAR_M1_53 & - & 64 & - & 256 & - \\
\hline RAR_M1_54 & - & 128 & - & - & - \\
\hline RAR_GA_57 & - & 32 & - & 16 & 64 \\
\hline RAR_M1_58 & - & 256 & - & - & \\
\hline RAR_M1_60 & 128 & - & 128 & - & 32 \\
\hline RAR_M1_61 & - & - & - & - & - \\
\hline RAR_GA_63 & 64 & 256 & - & - & \\
\hline RAR_GA_64 & - & - & 64 & & - \\
\hline RAR_M1_66 & 128 & - & - & 64 & 128 \\
\hline Ciprofloxacin & 1 & 2 & 0.5 & 0.5 & - \\
\hline Fluconazole & & & & & 2 \\
\hline
\end{tabular}

\subsubsection{Antioxidant Activity}

The antioxidant activity of the bacterial extracts was evaluated by their DPPH $(1,1-$ diphenyl-2-picrylhydrazyl) and ABTS (2,2-azino-bis-3-ethylbenzothiazoline-6-sulfonic acid) radical scavenging effects (Table 3). Antioxidant assays showed that nine extracts exhibited significant DPPH radical scavenging activity with $\mathrm{IC}_{50}$ values from $43.52 \pm 3.87$ to $88.32 \pm 4.13 \mu \mathrm{g} / \mathrm{mL}$ and eleven extracts exhibited significant ABTS radical scavenging activity with $\mathrm{IC}_{50}$ values from $56.29 \pm 4.61$ to $81.45 \pm 3.64 \mu \mathrm{g} / \mathrm{mL}$. Among them, eight extracts, i.e., RAR_GA_16, RAR_M1_23, RAR_GA_31, RAR_WA_32, RAR_GA_42, RAR_M1_44, RAR_M1_58, and RAR_M1_61 exhibited both DPPH and ABTS radical scavenging activities. 
Table 3. DPPH and ABTS radical scavenging activity of the bacterial extracts $\left(\mathrm{IC}_{50}, \mu \mathrm{g} / \mathrm{mL}\right)$.

\begin{tabular}{ccc}
\hline Strain ID & $\begin{array}{c}\text { DPPH Radical Scavenging } \\
\text { Activity }\end{array}$ & $\begin{array}{c}\text { ABTS Radical Scavenging } \\
\text { Activity }\end{array}$ \\
RAR_GA_12 & $>100$ & $>100$ \\
RAR_GA_16 & $72.24 \pm 5.26$ & $56.29 \pm 4.61$ \\
RAR_WA_18 & $>100$ & $>100$ \\
RAR_M1_23 & $61.23 \pm 3.26$ & $76.32 \pm 3.51$ \\
RAR_M1_26 & $>100$ & $>100$ \\
RAR_GA_31 & $72.36 \pm 3.95$ & $>100$ \\
RAR_WA_32 & $43.52 \pm 3.87$ & $66.43 \pm 5.25$ \\
RAR_M1_36 & $44.34 \pm 2.76$ & $64.51 \pm 3.26$ \\
RAR_M1_41 & $>100$ & $>100$ \\
RAR_GA_42 & $51.64 \pm 2.68$ & $76.29 \pm 3.75$ \\
RAR_M1_44 & $81.34 \pm 6.18$ & $77.35 \pm 5.82$ \\
RAR_M1_45 & $>100$ & $>100$ \\
RAR_M1_49 & $>100$ & $>100$ \\
RAR_WA_50 & $>100$ & $81.45 \pm 3.64$ \\
RAR_M1_53 & $>100$ & $>100$ \\
RAR_M1_54 & $>100$ & $>100$ \\
RAR_GA_57 & $>100$ & $59.43 \pm 2.68$ \\
RAR_M1_58 & $88.32 \pm 4.13$ & $67.48 \pm 2.46$ \\
RAR_M1_60 & $>100$ & $>100$ \\
RAR_M1_61 & $65.23 \pm 3.23$ & $77.21 \pm 3.24$ \\
RAR_GA_63 & $>100$ & $>100$ \\
RAR_GA_64 & $>100$ & $>100$ \\
RAR_M1_66 & $>100$ & $71.35 \pm 3.57$ \\
Ascorbic acid & $29.14 \pm 4.27$ & $26.35 \pm 3.24$ \\
\hline
\end{tabular}

2.2.3. $\alpha$-Amylase, $\alpha$-Glucosidase, and Xanthine Oxidase Inhibitory Activities

The inhibitory effects of the bacterial extracts against several enzymes related to diabetes and gout, i.e., $\alpha$-amylase, $\alpha$-glucosidase, and xanthine oxidase, also were evaluated (Table 4). Bioassays showed that eleven extracts exhibited significant $\alpha$-amylase inhibitory activity with $\mathrm{IC}_{50}$ values from $33.51 \pm 4.62$ to $131.36 \pm 5.41 \mu \mathrm{g} / \mathrm{mL}$, and nine extracts exhibited significant $\alpha$-glucosidase inhibitory activity with $\mathrm{IC}_{50}$ values from $53.68 \pm 3.12$ to $193.44 \pm 6.73 \mu \mathrm{g} / \mathrm{mL}$. Among them, six extracts exhibited both $\alpha$-amylase and $\alpha$-glucosidase inhibitory activities, such as RAR_GA_16, RAR_GA_31, RAR_WA_32, RAR_M1_44, RAR_M1_49, and RAR_GA_57. Notably, several extracts exhibited better $\alpha$-amylase and $\alpha$-glucosidase inhibitory activities than the positive control acarbose $\left(\mathrm{IC}_{50}=89.34 \pm 3.61\right.$ and $217.46 \pm 6.38$ for $\alpha$-amylase and $\alpha$-glucosidase, respectively $)$. Regarding the $\mathrm{XO}$ enzyme, ten extracts exhibited significant $\mathrm{XO}$ inhibitory activity with $\mathrm{IC}_{50}$ values from $54.57 \pm 2.53$ to $94.36 \pm 4.74 \mu \mathrm{g} / \mathrm{mL}$.

\subsubsection{Cytotoxic Activity}

The cytotoxic activity of the bacterial extracts against three cancer cell lines, i.e., MCF7 (human breast carcinoma), A549 (human lung carcinoma), and HeLa (human cervix carcinoma), are shown in Table 5. Cytotoxic bioassays showed that six extracts exhibited significant cytotoxic activity against the cell line MCF-7 with $\mathrm{IC}_{50}$ values from $36.48 \pm 2.63$ to $83.24 \pm 4.51 \mu \mathrm{g} / \mathrm{mL}$, four extracts exhibited significant cytotoxic activity against the cell line A549 with $\mathrm{IC}_{50}$ values from $21.52 \pm 3.22$ to $89.53 \pm 5.31 \mu \mathrm{g} / \mathrm{mL}$, and three extracts exhibited significant cytotoxic activity against the cell line A549 with $\mathrm{IC}_{50}$ values from $41.27 \pm 3.42$ to $97.53 \pm 5.31 \mu \mathrm{g} / \mathrm{mL}$. Of these, two extracts exhibited cytotoxic activity against two cell lines, i.e., RAR_WA_32 and RAR_WA_50, and two extracts exhibited cytotoxic activity against all three tested cell lines, i.e., RAR_GA_16 and RAR_M1_44. 
Table 4. $\alpha$-Amylase, $\alpha$-glucosidase, and xanthine oxidase inhibitory activities of the bacterial extracts $\left(\mathrm{IC}_{50}, \mu \mathrm{g} / \mathrm{mL}\right)$.

\begin{tabular}{cccc}
\hline Strain ID & $\begin{array}{c}\alpha \text {-Amylase } \\
\text { Inhibition }\end{array}$ & $\begin{array}{c}\alpha \text {-Glucosidase } \\
\text { Inhibition }\end{array}$ & XO Inhibition \\
\hline RAR_GA_12 & $>200$ & $74.26 \pm 4.12$ & $>100$ \\
RAR_GA_16 & $73.27 \pm 3.45$ & $85.73 \pm 5.26$ & $94.36 \pm 4.74$ \\
RAR_WA_18 & $>200$ & $>200$ & $54.57 \pm 2.53$ \\
RAR_M1_23 & $131.36 \pm 5.41$ & $>200$ & $>100$ \\
RAR_M1_26 & $>200$ & $73.64 \pm 3.78$ & $>100$ \\
RAR_GA_31 & $64.42 \pm 3.35$ & $81.32 \pm 4.42$ & $>100$ \\
RAR_WA_32 & $33.51 \pm 4.62$ & $53.68 \pm 3.12$ & $74.38 \pm 3.81$ \\
RAR_M1_36 & $51.67 \pm 2.98$ & $>200$ & $75.36 \pm 3.76$ \\
RAR_M1_41 & $>200$ & $0>200$ & $>100$ \\
RAR_GA_42 & $>200$ & $>200$ & $82.35 \pm 4.13$ \\
RAR_M1_44 & $86.38 \pm 5.39$ & $94.14 \pm 5.28$ & $64.43 \pm 4.56$ \\
RAR_M1_45 & $>200$ & $>200$ & $>100$ \\
RAR_M1_49 & $58.34 \pm 2.47$ & $55.16 \pm 4.39$ & $>100$ \\
RAR_WA_50 & $77.64 \pm 3.68$ & $>200$ & $>100$ \\
RAR_M1_53 & $>200$ & $>200$ & $74.38 \pm 3.81$ \\
RAR_M1_54 & $>200$ & $>200$ & $>100$ \\
RAR_GA_57 & $83.45 \pm 4.25$ & $57.38 \pm 3.42$ & $>100$ \\
RAR_M1_58 & $68.34 \pm 3.42$ & $>200$ & $71.44 \pm 3.67$ \\
RAR_M1_60 & $>200$ & $>200$ & $>100$ \\
RAR_M1_61 & $>200$ & $193.44 \pm 6.73$ & $>100$ \\
RAR_GA_63 & $128.34 \pm 6.32$ & $>200$ & $>100$ \\
RAR_GA_64 & $>200$ & $>200$ & - \\
RAR_M1_66 & $>200$ & $>200$ & $6.12 \pm 0.61$ \\
Acarbose & $89.34 \pm 3.61$ & - &
\end{tabular}

Table 5. Cytotoxic activity of bacterial extracts against cancer cell lines $\left(\mathrm{IC}_{50}, \mu \mathrm{g} / \mathrm{mL}\right)$.

\begin{tabular}{cccc}
\hline Strain ID & MCF-7 & A549 & Hela \\
\hline RAR_GA_12 & $>100$ & $>100$ & $>100$ \\
RAR_GA_16 & $36.48 \pm 2.63$ & $89.53 \pm 5.31$ & $41.27 \pm 3.42$ \\
RAR_WA_18 & $>100$ & $>100$ & $>100$ \\
RAR_M1_23 & $>100$ & $>100$ & $>100$ \\
RAR_M1_26 & $>100$ & $>100$ & $>100$ \\
RAR_GA_31 & $>100$ & $>100$ & $>100$ \\
RAR_WA_32 & $>100$ & $21.52 \pm 3.22$ & $57.67 \pm 4.75$ \\
RAR_M1_36 & $83.24 \pm 4.51$ & $>100$ & $>100$ \\
RAR_M1_41 & $>100$ & $>100$ & $>100$ \\
RAR_GA_42 & $53.17 \pm 2.84$ & $51.32 \pm 4.21$ & $>7.53 \pm 5.31$ \\
RAR_M1_44 & $61.32 \pm 3.22$ & $>100$ & $>100$ \\
RAR_M1_45 & $>100$ & $>100$ & $>100$ \\
RAR_M1_49 & $>100$ & $36.48 \pm 2.32$ & $>100$ \\
RAR_WA_50 & $61.32 \pm 3.22$ & $>100$ & $>100$ \\
RAR_M1_53 & $>100$ & $>100$ & $>100$ \\
RAR_M1_54 & $>100$ & $>100$ & $>100$ \\
RAR_GA_57 & $77.36 \pm 2.31$ & $>100$ & $>100$ \\
RAR_M1_58 & $>100$ & $>100$ & $>100$ \\
RAR_M1_60 & $>100$ & $>100$ & $>100$ \\
RAR_M1_61 & $>100$ & $>100$ & $>100$ \\
RAR_GA_63 & $>100$ & $>100$ & $>100$ \\
RAR_GA_64 & $>100$ & $>100$ & $3.56 \pm 0.62$ \\
RAR_M1_66 & $>100$ & $2.47 \pm 0.36$ & \\
Camptothecin & $4.75 \pm 0.41$ & & \\
\hline
\end{tabular}




\subsection{Volatile Chemical Composition of the Endophytic Bacterial Extracts by GC-MS}

The bioassays revealed that the ethyl acetate extracts of the three endophytic strains Bacillus sp. RAR_GA_16, Rossellomorea vietnamensis RAR_WA_32, and Bacillus sp. RAR_M1_44 displayed the most potential pharmacological properties. Thus, the composition of volatile organic compounds present in their ethyl acetate extracts was investigated by GC-MS analyses. The GC-MS analysis identified 29, 78, and 42 peaks of VOCs from the extract of the bacterial strains RAR_GA_16, RAR_WA_32, and RAR_M1_44, respectively (Supplementary Tables S1S3). By comparing with the spectra databases of the known compounds, 7 compounds were identified in the extract of Bacillus sp. RAR_GA_16 (Table 6) including hexahydro-pyrrolo[1,2a]pyrazine-1,4-dione (13.15\%), 3-isobutylhexahydropyrrolo[1,2-a]pyrazine-1,4-dione (8.15\%), palmitic acid $(2.09 \%)$, diisooctyl phthalate $(6.90 \%)$, linoleic acid (1.57\%), 9(E)-octadecenoic acid $(9.17 \%)$, and stearic acid $(3.02 \%), 26$ compounds were identified from the extract of Rossellomorea vietnamensis RAR_WA_32 with the major components including dioctyl phthalate (58.09\%), palmitic acid (5.56\%), 3-isobutylhexahydropyrrolo[1,2-a]pyrazine-1,4-dione (4.56\%) stearic acid (3.55\%), benzeneacetic acid (1.92\%), and 3-benzyl-hexahydro-pyrrolo[1,2-a]pyrazine-1,4-dione (1.875), and 6 compounds were identified from the extract of Bacillus sp. RAR_M1_44 including palmitic acid $(0.51 \%),(Z, Z)-9,12$-octadecadienoic acid $(0.33 \%)$, 6-octadecenoic acid (1.93\%), stearic acid (0.34\%), and 1,2-benzenedicarboxylic acid (0.99\%) (Table 6). Interestingly, several compounds were found in the extracts of all three bacterial strains Bacillus sp. RAR_GA_16, Rossellomorea vietnamensis RAR_WA_32, and Bacillus sp. RAR_M1_44 (i.e., palmitic acid and stearic acid); however, the quantities of the compounds varied among the extracts.

Table 6. The volatile chemical composition of the bacterial extracts.

\begin{tabular}{|c|c|c|c|c|c|c|}
\hline No. & Composition & $\mathrm{RT} / \mathrm{min}$ & $\begin{array}{l}\text { Matching } \\
\text { Quality }\end{array}$ & Quantity (\%) & $\begin{array}{l}\text { Biological } \\
\text { Activity }\end{array}$ & Refs. \\
\hline \multicolumn{7}{|c|}{ Bacillus sp. RAR_GA_16 } \\
\hline 1 & $\begin{array}{l}\text { Hexahydro-pyrrolo[1,2-a]pyrazine- } \\
\text { 1,4-dione }\end{array}$ & 18.830 & 96 & 13.15 & $\begin{array}{l}\text { Antimicrobial, } \\
\text { antioxidant }\end{array}$ & {$[28,29]$} \\
\hline 2 & $\begin{array}{l}\text { 3-Isobutylhexahydropyrrolo[1,2- } \\
\text { a]pyrazine-1,4-dione }\end{array}$ & 21.125 & 91 & 8.15 & $\begin{array}{c}\text { Antimicrobial, } \\
\text { nematicidal, } \\
\text { and } \\
\text { anti-mutagenic }\end{array}$ & {$[30-32]$} \\
\hline 3 & Palmitic acid & 21.413 & 98 & 2.09 & Antibacterial & [33] \\
\hline 4 & Diisooctyl phthalate & 22.022 & 95 & 6.90 & $\begin{array}{l}\text { Antibacterial, } \\
\text { antifungal }\end{array}$ & {$[34,35]$} \\
\hline 5 & Linoleic acid & 23.454 & 99 & 1.57 & Antibacterial & {$[33,36]$} \\
\hline 6 & $9(E)$-Octadecenoic acid & 23.517 & 99 & 9.17 & Antibacterial & [37] \\
\hline 7 & Stearic acid & 23.763 & 98 & 3.02 & Antibacterial & [33] \\
\hline \multicolumn{7}{|c|}{ Rossellomorea vietnamensis RAR_WA_32 } \\
\hline 1 & Benzyl alcohol & 8.045 & 96 & 0.12 & $\begin{array}{l}\text { Antibacterial } \\
\text { Antioxidant }\end{array}$ & {$[38,39]$} \\
\hline 2 & 2,3,5,6-Tetramethylpyrazine & 9.494 & 91 & 0.16 & $\begin{array}{l}\text { anticancer, anti- } \\
\text { inflammatory }\end{array}$ & {$[40-42]$} \\
\hline 3 & Phenylethyl alcohol & 10.225 & 97 & 0.16 & Antibacterial & {$[43,44]$} \\
\hline 4 & Benzoic acid & 11.783 & 94 & 0.25 & Antimicrobial & [45] \\
\hline 5 & Methyl phenylacetate & 12.022 & 94 & 0.19 & Antimicrobial & {$[46,47]$} \\
\hline 6 & Benzeneacetic acid & 14.193 & 94 & 1.92 & Antimicrobial & {$[48,49]$} \\
\hline 7 & Benzenepropanoic acid & 16.367 & 96 & 0.38 & Antimicrobial & {$[50]$} \\
\hline
\end{tabular}


Table 6. Cont.

\begin{tabular}{|c|c|c|c|c|c|c|}
\hline No. & Composition & $\mathrm{RT} / \mathrm{min}$ & $\begin{array}{l}\text { Matching } \\
\text { Quality }\end{array}$ & Quantity (\%) & $\begin{array}{l}\text { Biological } \\
\text { Activity }\end{array}$ & Refs. \\
\hline 8 & Docosane & 20.327 & 91 & 0.09 & - & - \\
\hline 9 & 2,4-Di-tert-butylphenol & 20.775 & 97 & 0.3 & $\begin{array}{l}\text { Antifungal, } \\
\text { antioxidant }\end{array}$ & {$[51,52]$} \\
\hline 10 & $\begin{array}{c}\text { Hexahydropyrrolo[1,2-a]pyrazine- } \\
\text { 1,4-dione }\end{array}$ & 26.144 & 96 & 0.38 & $\begin{array}{l}\text { Antibacterial, } \\
\text { antioxidant }\end{array}$ & {$[28,29]$} \\
\hline 11 & Myristic acid & 26.351 & 94 & 0.08 & Antibacterial & {$[53,54]$} \\
\hline 12 & Methyl 13-methylmyristate & 26.911 & 97 & 0.12 & - & - \\
\hline 13 & 12-Methyltetradecanoic acid & 27.078 & 94 & 0.23 & $\begin{array}{l}\text { Antifungal, } \\
\text { anticancer }\end{array}$ & {$[55,56]$} \\
\hline 14 & Pentadecanoic acid & 27.717 & 98 & 0.64 & - & - \\
\hline 15 & Methyl palmitate & 29.702 & 95 & 0.76 & $\begin{array}{l}\text { Antibacterial, } \\
\text { anti- } \\
\text { inflammatory }\end{array}$ & {$[57,58]$} \\
\hline 16 & $\begin{array}{l}\text { 3-Isobutylhexahydropyrrolo[1,2- } \\
\text { a]pyrazine-1,4-dione }\end{array}$ & 29.836 & 95 & 4.56 & $\begin{array}{l}\text { Antimicrobial, } \\
\text { nematicidal, } \\
\text { and } \\
\text { anti-mutagenic }\end{array}$ & {$[30-32]$} \\
\hline 17 & Palmitic acid & 30.482 & 99 & 5.56 & Antibacterial & {$[33]$} \\
\hline 18 & Methyl 14-methylhexadecanoate & 31.084 & 92 & 0.18 & - & - \\
\hline 19 & 11-Octadecenoic methyl ester & 33.090 & 95 & 0.08 & - & - \\
\hline 20 & Methyl stearate & 33.458 & 96 & 0.14 & - & - \\
\hline 21 & 9-Octadecenoic acid & 33.755 & 98 & 0.79 & Antibacterial & [59] \\
\hline 22 & Stearic acid & 34.135 & 99 & 3.55 & Antibacterial & [33] \\
\hline 23 & $\begin{array}{l}\text { 1,3,3a,6,7,9a-Hexahydro-cis- } \\
\text { cycloocta[c]furan }\end{array}$ & 35.701 & 90 & 0.23 & - & - \\
\hline 24 & Cyclo(phe-pro) & 36.941 & 90 & 0.15 & $\begin{array}{l}\text { Antimicrobial, } \\
\text { anticancer }\end{array}$ & {$[60-62]$} \\
\hline 25 & $\begin{array}{l}\text { 3-Benzylhexahydropyrrolo[1,2- } \\
\text { a]pyrazine-1,4-dione }\end{array}$ & 37.704 & 95 & 1.87 & $\begin{array}{l}\text { Anti-biofilm, } \\
\text { anti-quorum } \\
\text { sensing }\end{array}$ & [63] \\
\hline 26 & Dioctyl phthalate & 40.507 & 91 & 58.09 & $\begin{array}{l}\text { Antibacterial, } \\
\text { tyrosinase } \\
\text { inhibitory }\end{array}$ & [64-66] \\
\hline \multicolumn{7}{|c|}{ Bacillus sp. RAR_M1_44 } \\
\hline 1 & Palmitic acid & 21.416 & 97 & 0.51 & $\begin{array}{l}\text { Antimicrobial, } \\
\text { antioxidant }\end{array}$ & [33] \\
\hline 2 & 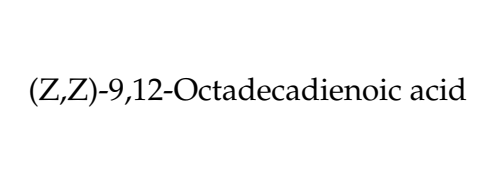 & 23.459 & 99 & 0.33 & $\begin{array}{c}\text { Anti- } \\
\text { inflammatory, } \\
\text { nematicidal, } \\
\text { and hepatopro- } \\
\text { tective }\end{array}$ & [67] \\
\hline 3 & cis-Vaccenic acid & 23.521 & 99 & 1.93 & $\begin{array}{l}\text { Antibacterial, } \\
\text { hypolipidemic }\end{array}$ & [68] \\
\hline 4 & Stearic acid & 23.764 & 99 & 0.34 & $\begin{array}{l}\text { Antibacterial, } \\
\text { antifungal }\end{array}$ & [33] \\
\hline 5 & 1,2-Benzenedicarboxylic acid & 23.978 & 90 & 0.99 & $\begin{array}{l}\text { Antifungal, } \\
\text { anticancer }\end{array}$ & {$[33,36]$} \\
\hline
\end{tabular}

Compounds with minimum match quality of $90 \%$.

2.4. Genome Sequencing, Assembly, and Annotation of Biosynthesis Gene Clusters of Secondary Metabolites

In order to discover the biosynthesis gene clusters (BGCs) of secondary metabolites of the isolated bacteria, we sequenced their genomes and annotated BGCs of secondary metabolites from the genome data. The genomic features of three bacterial strains Bacillus sp. RAR_GA_16, Rossellomorea vietnamensis RAR_WA_32, and Bacillus sp. RAR_M1_44 are shown in Table 7. The size of the bacterial genomes was from 3,768,026 to 4,494,267 bp with GC contents from 40.69 to $44.09 \%$. Assembly results showed that completion 
of the genomes was from 96.6 to $99.8 \%$, with the maximum contig length from 770,470 to 2,585,281 bp and N50 contig length from 501,856 to 2,582,281 bp. Annotation of the genomes by Prokka showed that the genomes contain 3807-4610 coding sequences (CDSs), 22-27 rRNA, 81-111 tRNA, and 0-1 tmRNA.

Table 7. Genomic features of the bacterial endophytes.

\begin{tabular}{cccc}
\hline Genomic Features & RAR_GA_16 & RAR_WA_32 & RAR_M1_44 \\
\hline Size of the genome assembly (bp) & $4,394,636$ & $4,494,267$ & $3,768,026$ \\
GC content (\%) & 40.69 & 44.09 & 41.38 \\
Contigs & 22 & 4 & 5 \\
Maximum Contig Length (bp) & 770,470 & $2,474,194$ & $2,582,281$ \\
N50 Contig Length (bp) & 501,856 & $2,474,194$ & $2,582,281$ \\
CDS & 4610 & 4529 & 3807 \\
rRNA & 27 & 22 & 24 \\
tRNA & 89 & 111 & 81 \\
tmRNA & 1 & 0 & 1 \\
Complete genome (\%) & 96.6 & 99.5 & 99.8 \\
\hline
\end{tabular}

The genomes were analyzed for the presence of secondary metabolite biosynthetic gene clusters (BGCs) using antiSMASH. Annotation results showed that the genome of Bacillus sp. RAR_GA_16 contains 22 BGCs, including clusters related to the biosynthesis of linear azol(in)e-containing peptide (LAP), bacteriocin, lassopeptide, siderophore, type III polyketide (T3PKS), terpene, and several unknown compounds. Several BGCs shared their identity to the biosynthesis clusters of known compounds in Minimum Information about a Biosynthetic Gene cluster (MIBiG) database, such as paeninodin, butirosin A, butirosin B, carotenoid, thaxteramide $C$, and fengycin with the identities of 4-80\% (Table 8).

Table 8. Predicted biosynthesis gene clusters of secondary metabolites from the bacterial genomes.

\begin{tabular}{|c|c|c|c|c|c|}
\hline Cluster & Length & Types & $\begin{array}{l}\text { Most Similar } \\
\text { Known Cluster }\end{array}$ & MIBiG BGC-ID & Similarity \\
\hline \multicolumn{6}{|c|}{ Bacillus sp. RAR_GA_16 } \\
\hline Cluster 1 & 21,787 & LAP, bacteriocin & - & - & - \\
\hline Cluster 2 & 14,207 & Lassopeptide & Paeninodin & BGC0001356 & $80 \%$ \\
\hline Cluster 3 & 28,959 & Siderophore & - & - & - \\
\hline Cluster 4 & 37,598 & T3PKS & - & - & - \\
\hline Cluster 5 & 21,821 & Terpene & $\begin{array}{c}\text { Butirosin } \\
\text { A/butirosin B }\end{array}$ & BGC0000693 & $7 \%$ \\
\hline Cluster 6 & 20,837 & Terpene & Carotenoid & BGC0000645 & $50 \%$ \\
\hline Cluster 7 & 24,479 & - & S-layer glycan & BGC0000796 & $14 \%$ \\
\hline Cluster 8 & 15,240 & - & Thaxteramide C & BGC0002025 & $7 \%$ \\
\hline Cluster 9 & 4858 & - & Fengycin & BGC0001095 & $20 \%$ \\
\hline Cluster 10 & 8288 & - & $\begin{array}{c}\text { Capsular } \\
\text { polysaccharide }\end{array}$ & BGC0000758 & $4 \%$ \\
\hline Cluster 11 & 11,638 & - & $\begin{array}{c}- \\
\end{array}$ & - & - \\
\hline Cluster 12 & 6299 & - & - & - & - \\
\hline Cluster 13 & 3468 & - & - & - & - \\
\hline Cluster 14 & 15,194 & - & - & - & - \\
\hline Cluster 15 & 9297 & - & - & - & - \\
\hline Cluster 16 & 15,779 & - & - & - & - \\
\hline Cluster 17 & 11,263 & - & - & - & - \\
\hline Cluster 18 & 9795 & - & - & - & - \\
\hline Cluster 19 & 9448 & - & - & - & - \\
\hline Cluster 20 & 16,830 & - & - & - & - \\
\hline Cluster 21 & 7027 & - & - & - & - \\
\hline Cluster 22 & 5280 & - & - & - & - \\
\hline
\end{tabular}


Table 8. Cont.

\begin{tabular}{|c|c|c|c|c|c|}
\hline Cluster & Length & Types & $\begin{array}{c}\text { Most Similar } \\
\text { Known Cluster }\end{array}$ & MIBiG BGC-ID & Similarity \\
\hline \multicolumn{6}{|c|}{ Rossellomorea vietnamensis RAR_WA_32 } \\
\hline Cluster 1 & 21,869 & Terpene & $\begin{array}{c}\text { Pyxidicycline } \\
\text { A/pyxidicycline B }\end{array}$ & BGC0001940 & $6 \%$ \\
\hline Cluster 2 & 20,443 & Saccharide & A40926 & BGC0000289 & $3 \%$ \\
\hline Cluster 3 & 22,620 & Saccharide & Carotenoid & BGC0000645 & $33 \%$ \\
\hline Cluster 4 & 121,332 & Saccharide & S-layer glycan & BGC0000796 & $14 \%$ \\
\hline Cluster 5 & 50,793 & $\begin{array}{c}\text { Fatty_acid, } \\
\text { saccharide, terpene }\end{array}$ & Carotenoid & BGC0000645 & $50 \%$ \\
\hline Cluster 6 & 19,105 & LAP, RiPP-like & - & - & - \\
\hline Cluster 7 & 20,392 & Saccharide & - & - & - \\
\hline Cluster 8 & 70,095 & Saccharide,T3PKS & - & - & - \\
\hline Cluster 9 & 29,497 & Saccharide & - & - & - \\
\hline Cluster 10 & 21,005 & Fatty_acid & - & - & - \\
\hline Cluster 11 & 30,306 & Saccharide & - & - & - \\
\hline Cluster 12 & 34,473 & Saccharide & - & - & - \\
\hline Cluster 13 & 21,368 & Saccharide & - & - & - \\
\hline Cluster 14 & 24,846 & Saccharide & - & - & - \\
\hline Cluster 15 & 30,807 & Saccharide & - & - & - \\
\hline Cluster 16 & 35,649 & Saccharide & - & - & - \\
\hline \multicolumn{6}{|c|}{ Bacillus sp. RAR_M1_44 } \\
\hline Cluster 1 & 28,756 & $\begin{array}{l}\text { Siderophore, } \\
\text { terpene }\end{array}$ & Carotenoid & BGC0000645 & $50 \%$ \\
\hline Cluster 2 & 28,411 & Betalactone & Fengycin & BGC0001095 & $53 \%$ \\
\hline Cluster 3 & 83,725 & NRPS & Lichenysin & BGC0000868 & $85 \%$ \\
\hline Cluster 4 & 8013 & Other & Teichuronic acid & BGC0001184 & $75 \%$ \\
\hline Cluster 5 & 41,421 & Other & Bacilysin & BGC0000381 & $85 \%$ \\
\hline Cluster 6 & 21,877 & Terpene & - & - & - \\
\hline Cluster 7 & 41,097 & T3PKS & - & - & - \\
\hline Cluster 8 & 26,707 & Bacteriocin & & & \\
\hline Cluster 9 & 32,416 & Betalactone & & & \\
\hline Cluster 10 & 15,922 & - & - & - & - \\
\hline Cluster 11 & 5255 & - & - & - & - \\
\hline Cluster 12 & 13,700 & - & - & - & - \\
\hline Cluster 13 & 8838 & - & - & - & - \\
\hline Cluster 14 & 16,010 & - & - & - & - \\
\hline Cluster 15 & 3876 & - & - & - & - \\
\hline Cluster 16 & 9767 & - & - & - & - \\
\hline
\end{tabular}

Regarding the genome of Rossellomorea vietnamensis RAR_WA_32, annotation results showed that the genome contains 16 BGCs, including clusters related to the biosynthesis of terpene, LAP, type III polyketide, fatty acid, other unspecified ribosomally synthesised and post-translationally modified peptide product (RiPP-like), and saccharide (likely from primary metabolism). Several BGCs shared their identity to known biosynthesis clusters in the MIBiG database, such as pyxidicycline A, pyxidicycline B, A40926, and carotenoid with the identities of $3-50 \%$ (Table 8 ).

In the case of the genome of Bacillus sp. RAR_M1_44, it contains 16 BGCs, including clusters related to the biosynthesis of terpene, siderophore, betalactone, nonribosomal peptide (NRP), type III polyketide, bacteriocin, others and unknown compounds. Several BGCs shared their identity to the biosynthesis clusters of known compounds in the MIBiG database, such as carotenoid, fengycin, lichenysin, teichuronic acid, and bacilysin with the identities of $50-85 \%$ (Table 8 ). The gene structures of BCGs having the identities $>70 \%$ with known compound clusters in the MIBiG database are shown in Figure 3. 

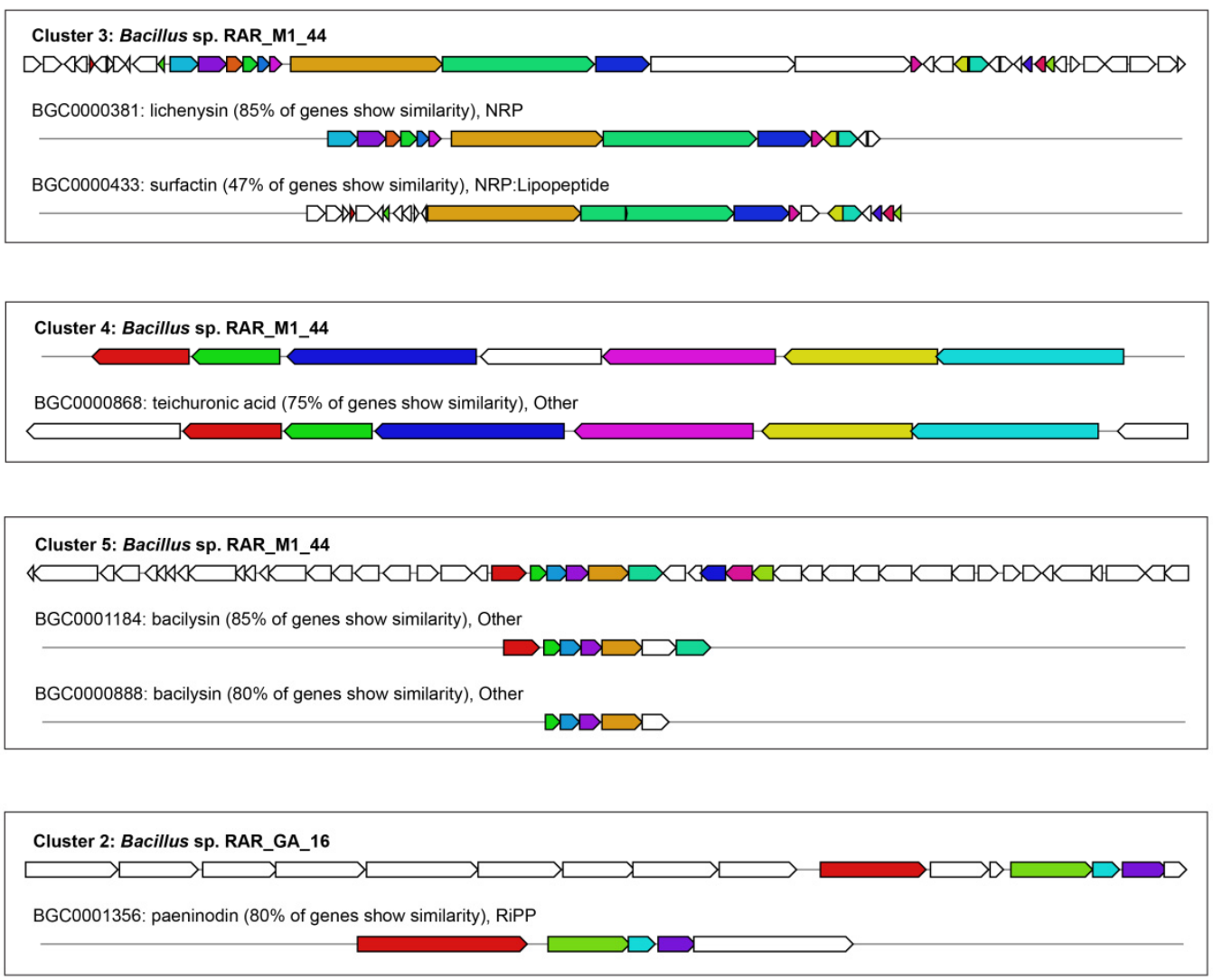

Figure 3. Similarity of predicted BGCs from genomes and the MIBiG database.

The BGCs predicted from the genomes were analyzed by the Biosynthetic Genes Similarity Clustering and Prospecting Engine (BIG-SCAPE) to group the homologous BGCs into gene cluster families (GCFs), which can be responsible for the production of the same compound or similar compounds. Interestingly, no GCF was found between the BCGs from the genomes as well as between the BCGs from the genomes and the BGCs from the MIBiG database.

The ketide synthase (KS) and condensation (C) domains are the most conserved catalytic domains of polyketide synthase (PKS) and non-ribosomal peptide synthetase (NRPS) genes, respectively. Therefore, in order to predict the secondary metabolites of polyketides and non-ribosomal peptides, KS- and C- domains from the genomes were analyzed by the Natural Product Domain Seeker (NapDos). Analyses showed that the genomes of Rossellomorea vietnamensis RAR_WA_32 and Bacillus sp. RAR_M1_44 contain only KS domains without $C$ domains (Table 9). These KS sequences shared low identities with the KS sequences of the known products such as kirromycin, mycinamicin, rifamycin, amphotericin, spinosad, and dynemicin (26-45\% identity), except for fatty acids with $71-76 \%$ identity. Regarding the genome of Bacillus sp. RAR_GA_16, three KS domains and eleven $C$ domains were found in its genome (Table 9). These sequences shared low identities with the domain sequences of the known products such as mycocerosic acid, lychenicin, surfactin, and actinomycin (22-58\%), except for fatty acid ( $84 \%)$. 
Table 9. Predicted pathway products of KS and C domains from the bacterial genomes.

\begin{tabular}{|c|c|c|c|c|}
\hline $\begin{array}{c}\mathrm{KS} / \mathrm{C} \\
\text { Domain ID }\end{array}$ & Database Match ID & $\begin{array}{c}\text { Percent Identity } \\
(\%)\end{array}$ & E-Value & $\begin{array}{l}\text { Pathway } \\
\text { Product }\end{array}$ \\
\hline \multicolumn{5}{|c|}{ Bacillus sp. RAR_GA_16 } \\
\hline KS1 & AmphI_Q93NX9_1mod & 42 & $4 \mathrm{e}-7$ & Amphotericin \\
\hline KS2 & SpnB_Q9ALM5_1KSB & 35 & $2 \mathrm{e}-7$ & Spinosad \\
\hline KS3 & FabF_Bacillus_FAS & 76 & 0 & Fatty acid \\
\hline KS4 & DynE_AAN79725_ene10 & 45 & $9 e-6$ & Dynemicin \\
\hline \multicolumn{5}{|c|}{ Rossellomorea vietnamensis RAR_WA_32 } \\
\hline KS1 & KirAI_CAN89631_2T & 40 & $5 e-7$ & Kirromycin \\
\hline KS2 & FabB_Streptomyces_FAS & 41 & $3 e-6$ & Fatty acid \\
\hline KS3 & MycAIII_Q83WE8_2KSB & 44 & $1 e-6$ & Mycinamicin \\
\hline KS4 & FabF_Bacillus_FAS & 71 & 0.0 & Fatty acid \\
\hline KS5 & RifB_O52545_1mod & 26 & $1 e-6$ & Rifamycin \\
\hline \multicolumn{5}{|c|}{ Bacillus sp. RAR_M1_44 } \\
\hline KS1 & Myca_YP881572_1KSB & 40 & $2 e-6$ & $\begin{array}{c}\text { Mycocerosic } \\
\text { acid }\end{array}$ \\
\hline KS2 & FabF_Bacillus_FAS & 48 & $1 \mathrm{e}-108$ & Fatty acid \\
\hline KS3 & FabF_Bacillus_FAS & 84 & 0.0 & Fatty acid \\
\hline $\mathrm{C} 1$ & Liche1_C1_start & 51 & $3 e-135$ & Lychenicin \\
\hline $\mathrm{C} 2$ & Liche1_C1_start & 24 & $4 e-25$ & Lychenicin \\
\hline $\mathrm{C} 3$ & Liche1_C1_start & 22 & $6 e-18$ & Lychenicin \\
\hline $\mathrm{C} 4$ & Surfa5_C1_DCL & 57 & $5 e-151$ & Surfactin \\
\hline C5 & Surfa5_C1_DCL & 24 & $2 e-24$ & Surfactin \\
\hline C6 & Surfa5_C1_DCL & 22 & $3 e-12$ & Surfactin \\
\hline $\mathrm{C} 7$ & Surfa5_C1_DCL & 24 & $3 e-8$ & Surfactin \\
\hline $\mathrm{C} 8$ & Liche3_C1_DCL & 58 & $5 e-151$ & Lychenicin \\
\hline C9 & Act3_C3_LCL & 39 & $6 e-86$ & Actinomycin \\
\hline C10 & Act3_C3_LCL & 23 & $2 e-20$ & Actinomycin \\
\hline C11 & Liche1_C3_LCL & 38 & $2 e-77$ & Lychenicin \\
\hline
\end{tabular}

\section{Discussion}

Mangrove plants harbour diverse endophytic bacteria and fungi. Reviews on mangrove plant-associated microbial secondary metabolites revealed that the secondary metabolites possess promising pharmacological properties [8-11]. Unsurprisingly, mangrove endophytic microbes have been attracting the considerable attention of pharmacological investigators because of their bioactive secondary metabolites, especially when their host plants are also used as native traditional/folk medicines [69]. Over the last decade, almost 1000 new natural products have been reported from mangrove associated microbes, of these, $\sim 850$ are derived from fungi and $\sim 120$ are derived from bacteria (the majority of them derived from endophytes) [10].

In the present study, twenty-three endophytic bacteria belonging to nine genera (i.e., Microbulbifer, Streptomyces, Bacillus, Rossellomorea, Micrococcus, Paracoccus, Microbacterium, Pseudovibrio, and Brevibacterium) were isolated from the fresh roots of R. apiculata. Previous investigations revealed that the genera Bacillus, Streptomyces, and Pseudovibrio have been isolated frequently from mangrove plants, whereas Microbulbifer, Micrococcus, Paracoccus, Microbacterium, and Brevibacterium have been isolated less frequently [10,70-73]. Genome analyses of the genera Bacillus, Streptomyces, and Pseudovibrio have reported that genomes of these genera contain versatile and diverse genes of metabolic pathways as well as genes linked to symbiosis and lifestyles, allowing for host switching and adapting to various environments [74-80].

The endophytic bacterial ethyl acetate extracts in the present study were evaluated for their potential pharmacological properties. The bioassays revealed that these endophytic bacteria may be promising sources of bioactive secondary metabolites because their extracts exhibited antimicrobial, cytotoxic, antioxidant, and enzyme inhibitory effects (i.e., $\alpha$-amylase, $\alpha$-glucosidase, and xanthine oxidase). These results are consistent with previous investigations of bioactive natural products from endophytic microorganisms 
with the mangrove plant $R$. apiculata. Rukachaisirikul et al. [81] isolated nineteen secondary compounds from the endophytic fungus Acremonium sp. PSU-MA70 from the plant $R$. apiculata. Among them, the two compounds 8-deoxytrichothecin and trichodermol exhibited antifungal activity against $C$. albicans and Cryptococcus neoformanns with MIC values of 16-64 $\mu \mathrm{g} / \mathrm{mL}$. Klaiklay et al. [82] reported the isolation of 7 antifungal compounds from the endophytic fungus Pestalotiopsis sp. PSU-MA69 from the plant R. apiculata, including pestalolide, (s)-penipratynolene, pestalotether A-B, pestheic acid, chloroisosulochrin dehydrate, and chloroisosulochrin, with MICs of 128-200 $\mu \mathrm{g} / \mathrm{mL}$. Zhou et al. [26] isolated three antimicrobial compounds, fusolanone A-B and fusaric acid, from the endophytic fungus Fusarium solani HDN15-410 from the plant $R$. apiculata. These compounds exhibited good antimicrobial activity against a wide range of pathogenic microbes, i.e., P. aeruginosa, Monilia albican, B. subtilis, and Vibrio parahaemolyticus, with MICs of $6.25-50 \mu \mathrm{g} / \mathrm{mL}$. Fan et al. [24] isolated nine anti-H1N1 viral indole-diterpenoids from the fungus Penicillium camemberti OUCMDZ-1492 around roots of $R$. apiculata with $\mathrm{IC}_{50}$ values of $6.6-77.9 \mu \mathrm{M}$. Chaeprasert et al. [25] isolated 1921 fungal endophytic strains from 10 mangrove plant species R. apiculata, R. mucronata, Ceriops decandra, Sonneratia alba, Lumnitzera littorea, Avicennia alba, Acanthus ilicifolius, Xylocarpus granatum, X. moluccensis, and Thespesia populneoides. Among them, the ethyl acetate extract of 22 strains exhibited antimicrobial activity against B. subtilis, E. coli, P. aeruginosa, and S. aureus with zone inhibition diameters of $10-40 \mathrm{~mm}$, and the ethyl acetate extract of 26 strains exhibited cytotoxic activity against the cancer cell lines A375 (malignant melanoma), HepG2 (liver hepatoblastoma), SW620 (colorectal adenocarcinoma), Jurkat (acute T cell leukemia), and KatoIII (gastric carcinoma). Most extracts inhibited the cancer cell lines with growth inhibition of $>60 \%$. Klaiklay et al. [83] isolated cytotoxic and antibacterial compounds from the endophytic fungus Phomopsis sp. PSU-MA214 from the plant R. apiculata. Compound (2R,3S)-7-ethyl-1,2,3,4-tetrahydro2,3,8-trihydroxy-6-methoxy-3-methyl-9,10-anthracendione exhibited cytotoxicity against the cancer cell line MCF-7 with $\mathrm{IC}_{50}$ of $27 \mu \mathrm{g} / \mathrm{mL}$ and antibacterial activity against S. aureus and methicillin-resistant $S$. aureus with MICs of $128 \mu \mathrm{g} / \mathrm{mL}$ and $64 \mu \mathrm{g} / \mathrm{mL}$, respectively, whereas compound phomonitroester exhibited cytotoxicity against the cancer cell line KB with $\mathrm{IC}_{50}$ of $43 \mu \mathrm{g} / \mathrm{mL}$. Klaiklay et al. [84] isolated an antimicrobial compound, tremulenolide A, from the endophytic fungus Flavodon flavus PSU-MA201 from the plant R. apiculata. The compound exhibited antimicrobial activity against $S$. aureus and $C$. neoformans with MICs of $128 \mu \mathrm{g} / \mathrm{mL}$.

The ethyl acetate extracts of three endophytic bacteria with the most potential biological properties, i.e., Bacillus sp. RAR_GA_16, Rossellomorea vietnamensis RAR_WA_32, and Bacillus sp. RAR_M1_44, were investigated for their volatile chemical components by GC-MS analyses. Interestingly, many compounds identified in the extracts of three endophytic bacteria have been reported to have biological activities (e.g., antibacterial, antifungal, antivirus, anticancer, anti-inflammatory, antioxidant, nematicidal, anti-quorum sensing, tyrosinase inhibitory, antibiofilm, and anti-mutagenic activities) by previous studies (Table 6). These GC-MS analyses support the biological activity evaluation results of the ethyl acetate extracts. Additionally, it is noted that the identified VOCs from the extracts only accounted for $44.05 \%, 80.98 \%$, and $4.10 \%$ of the total amount of VOCs in the extracts of Bacillus sp. RAR_GA_16, Rossellomorea vietnamensis RAR_WA_32, and Bacillus sp. RAR_M1_44, respectively, whereas many VOCs in the extracts were not identified by their low match quality with the spectra data of known compounds (Supplementary Tables S1-S3). This implies that many VOCs in the extracts may be undescribed or novel compounds.

In the present study, the genomes of three potential endophytic bacteria, i.e., Bacillus sp. RAR_GA_16, Rossellomorea vietnamensis RAR_WA_32, and Bacillus sp. RAR_M1_44, were also sequenced and the presence of BGCs of secondary metabolites in their genomes was discovered. Annotations using the antiSMASH found 16-22 BGCs, 3-5 KS domains, and $11 \mathrm{C}$ domains in the bacterial genomes. However, the majority of BGCs, KS- and C-domains shared low identity with BGC, KS- and C-sequences in the known product database. Additionally, the BIG-SCAPE analysis also indicated that no BGCs predicted 
from the genomes were grouped in GCFs of known products in the MIBiG database. These findings suggest that the BGCs of the endophytic bacteria may biosynthesize novel compounds or compounds for which their BGCs have not been known. Interestingly, no GCF was found between the BGCs predicted from the genomes, implying that the BGCs of the endophytic bacteria may biosynthesize dissimilar compounds. The obtained results in the present study indicate that these endophytic bacteria may be potential sources for the discovery of novel bioactive metabolites, and further investigations, e.g., fermentation and isolation of pure bioactive compounds, as well as heterologous expression of novel BGCs in appropriate expression hosts, will allow exploring and exploiting novel bioactive compounds as well as demonstrating their biosynthesis pathways.

\section{Materials and Methods}

\subsection{Plant Collection}

The mangrove plant Rhizophora apiculata (Figure 1) was collected from the mangrove forest of $\mathrm{Bu} \mathrm{Lu}$, Phu Loc district, Thua Thien Hue province, Vietnam, in March 2019. The plant sample was transported to the laboratory of the Department of Biotechnology, Mientrung Institute for Scientific Research, for the isolation of the endophytic bacteria.

\subsection{Isolation and Identification of the Endophytic Bacteria}

The fresh plant root systems were washed thoroughly with running water to remove soil particles. The root surfaces were then washed in sterile distilled water six times, soaked in $70 \%$ ethanol for $5 \mathrm{~min}$ followed by $5 \%$ sodium hypochlorite for $10 \mathrm{~min}$, and again in $70 \%$ ethanol for $60 \mathrm{~s}$ before washing in distilled water three times.

The root samples $(5 \mathrm{~g})$ were crushed in $5 \mathrm{~mL}$ of sterile distilled water. A total of $100 \mu \mathrm{L}$ of root solution was spread on M1 medium ( $1.0 \%$ soluble starch, $0.4 \%$ yeast extract, $0.2 \%$ peptone, $1.5 \%$ agar), Gause no. 1 medium $\left(2.0 \%\right.$ soluble starch, $0.1 \% \mathrm{KNO}_{3}, 0.05 \%$ $\mathrm{NaCl}, 0.05 \% \mathrm{~K}_{2} \mathrm{HPO}_{4}, 0.05 \% \mathrm{MgSO}_{4}, 0.001 \% \mathrm{FeSO}_{4}, 1.5 \%$ agar), and water agar (1.5\% agar in mangrove water). The plates were incubated at $30^{\circ} \mathrm{C}$ for $3-5$ days. After incubation, colonies with different morphological characteristics (e.g., color, shape, and consistency) were streaked on nutrient agar plates (NA, Himedia, Mumbai, India) to obtain the pure isolates [70].

The representative isolates with different morphotypes were identified by colony PCR [85]. In brief, for cell lysis, the culture broths $(2 \mathrm{~mL})$ were centrifuged at $14,000 \times g$ for $10 \mathrm{~min}$ and the obtained pellets were suspended in $50 \mu \mathrm{L}$ nuclease-free water. Subsequently, the cell suspension was stored at $-20^{\circ} \mathrm{C}$ for $2 \mathrm{~h}$, followed by incubation at $98^{\circ} \mathrm{C}$ for $10 \mathrm{~min}$. The 16S rRNA gene of the isolates was directly amplified with universal primers $27 \mathrm{f}$ (5'-AGAGTTTGATCCTGGCTCAG-3') and 1492r (5'-GGTTACCTTGTTACGACTT-3') [86] with the following PCR program: an initial denaturation at $94^{\circ} \mathrm{C}$ for $5 \mathrm{~min}$, followed by 30 cycles of denaturation at $94{ }^{\circ} \mathrm{C}$ for $1 \mathrm{~min}$, annealing at $56^{\circ} \mathrm{C}$ for $50 \mathrm{~s}$, amplification at $72{ }^{\circ} \mathrm{C}$ for $1.5 \mathrm{~min}$, and a final extension at $72{ }^{\circ} \mathrm{C}$ for $7 \mathrm{~min}$. The $16 \mathrm{~S}$ rRNA gene sequencing was carried by the ABI PRISM $3100^{\circledR}$ Genetic Analyzer (Applied Bioscience and Hitachi, Foster City, CA, USA). The sequences were quality checked, and low-quality regions were removed from the sequence ends using BioEdit software v.7.2.6.1. The quality-checked sequences of the isolates were compared to available sequences in the NCBI GenBank using the BLAST searching program with the megablast algorithm and the database nt. The sequences were aligned using the ClustalW algorithm and the phylogenetic tree of $16 \mathrm{~S}$ rRNA sequences was created by the neighbor joining algorithm with 1000 bootstraps using MEGA v.7.0.0.

\subsection{Preparation of Ethyl Acetate Extracts from the Culture Broths}

The endophytic bacterial strains were cultured in $500 \mathrm{~mL}$ nutrient broth (NB, Himedia, Mumbai, India) for 7 days at $37^{\circ} \mathrm{C}$ under the shaking condition at $150 \mathrm{rpm}$, and the cultures were then centrifuged at $10.000 \mathrm{rpm}$ for $10 \mathrm{~min}$. The cell-free supernatants were extracted with ethyl acetate (1:1 v/v, 5 times) overnight at room temperature, and the ethyl acetate 
extractions were then evaporated under the reduced pressure for $12-24 \mathrm{~h}$ at $50{ }^{\circ} \mathrm{C}$ ) to remove ethyl acetate and obtain the crude extracts.

\subsection{Pharmacological Properties of Bacterial Crude Extracts}

\subsubsection{Antimicrobial Activity}

Antimicrobial activity of the bacterial extracts was tested against five reference microbes obtained from Mientrung Institute for Scientific Research, Thua Thien Hue province, Vietnam, i.e., Staphylococcus aureus ATCC 25923, Enterococcus faecalis ATCC 29212, Escherichia coli ATCC 25922, Pseudomonas aegurinosa ATCC 27853, and Candida albicans ATCC 10231.

Minimum inhibitory concentrations (MICs) of the extracts against the reference microorganisms were determined using the broth microdilution method as described by Dat et al. [85]. Briefly, $100 \mu \mathrm{L}$ of the bacterial inoculum $\left(1 \times 10^{6} \mathrm{CFU} / \mathrm{mL}\right)$ was added to wells containing $100 \mu \mathrm{L}$ of the extracts at a range of different concentrations in 96-well plates. The plate was incubated at $37^{\circ} \mathrm{C}$ for $24 \mathrm{~h}$, the absorbance at $630 \mathrm{~nm}$ was then measured using ELx800 absorbance microplate reader (BioTek Instruments, Winooski, VT, USA). MICs of the antibacterial extracts were determined as the lowest concentration, at which there was no growth of the bacteria. For the yeast, $100 \mu \mathrm{L}$ of the inoculum (2 to $5 \times 10^{5} \mathrm{CFU} / \mathrm{mL}$ for yeast) was added to wells containing $100 \mu \mathrm{L}$ of the extracts at a range of different concentrations in 96-well plates. The plate was incubated at $28{ }^{\circ} \mathrm{C}$ for $48 \mathrm{~h}$. MICs of the anti-yeast extracts were determined as the lowest concentration, at which there was no growth of the yeast by the absorbance at $530 \mathrm{~nm}$ using an ELx800 absorbance microplate reader (BioTek Instruments, Winooski, VT, USA). The antibiotics ciprofloxacin and fluconazole were used as positive controls for the tested bacteria and yeast, respectively.

\subsubsection{Antioxidant Activity}

The antioxidant effect of the extracts was determined by DPPH and ABTS radical scavenging assays [70].

DPPH radical scavenging effect of the extracts was determined by measuring the decrease in absorbance of DPPH radical solution in the presence of the extracts. In brief, $10 \mu \mathrm{L}$ of extracts was added to $190 \mu \mathrm{L}$ of DPPH $(0.1 \mathrm{mg} / \mathrm{mL})$ in $96-w e l l$ plates. The solution was mixed for $1 \mathrm{~min}$ and incubated at room temperature for $30 \mathrm{~min}$, and the absorbance of the reaction mixture was then recorded at $517 \mathrm{~nm}$ using an ELx800 absorbance microplate reader (BioTek Instruments, Winooski, VT, USA). Ascorbic acid was used as a positive control. The DPPH radical scavenging activity was calculated as follows: DPPH scavenging activity $(\%)=100 \times[\mathrm{Ac}-(\mathrm{As}-\mathrm{Asb}) / \mathrm{Ac}]$. Where: Ac is the absorbance of the control (only DPPH solution), As is absorbance of sample (extract with DPPH), and Asb is the absorbance of the sample blank (extract without DPPH).

ABTS radical scavenging effect of the extracts was determined by measuring the decrease in absorbance of ABTS radical solution in the presence of the extracts. In brief, two solutions (ABTS $7 \mathrm{mM}$ and potassium persulfate $2.45 \mathrm{mM}$ ) were mixed and allowed to stand in the dark at room temperature for $16 \mathrm{~h}$ before use in order to produce ABTS radical solution. The ABTS radical solution was then diluted with ethanol to give an absorbance of $0.700 \pm 0.02$ at $734 \mathrm{~nm}$. Ten microliters of the extracts was added to $190 \mu \mathrm{L}$ of ABTS radical solution in 96-well plates. The mixture was incubated at room temperature for $10 \mathrm{~min}$, and the absorbance of the reaction was then recorded at $734 \mathrm{~nm}$ using ELx800 absorbance microplate reader (BioTek Instruments, Winooski, VT, USA). Ascorbic acid was used as a positive control. The ABTS radical scavenging activity was calculated as follows: ABTS scavenging activity $(\%)=100 \times[\mathrm{Ac}-(\mathrm{As}-\mathrm{Asb}) / \mathrm{Ac}]$. Where: Ac is the absorbance of the control (only ABTS solution), As is absorbance of sample (extract with ABTS), and Asb is the absorbance of the sample blank (extract without ABTS). 


\subsection{3. $\alpha$-Amylase and $\alpha$-Glucosidase Activities}

The $\alpha$-amylase (A8220, Sigma-Aldrich, St. Louis, MO, USA) enzyme inhibitory effect of the extracts was determined according to the described method by Dat et al. [70]. In brief, starch azure was suspended in $0.05 \mathrm{M}$ Tris- $\mathrm{HCl}$ buffer ( $\mathrm{pH}$ 6.9) containing $0.01 \mathrm{M}$ $\mathrm{CaCl}_{2}$, and the substrate solution was then boiled for $5 \mathrm{~min}$ and pre-incubated at $37^{\circ} \mathrm{C}$ for $5 \mathrm{~min}$. The reaction mixture consisting of $50 \mu \mathrm{L}$ of the extract was incubated with $50 \mu \mathrm{L}$ of the substrate solution and $25 \mu \mathrm{L}$ of $\alpha$-amylase solution in Tris- $\mathrm{HCl}$ buffer $(2 \mathrm{U} / \mathrm{mL})$ in 96-well plates at $37^{\circ} \mathrm{C}$ for $10 \mathrm{~min}$. The reaction was stopped by adding $75 \mu \mathrm{L}$ of acetic acid $50 \%$, and the reaction solution was then centrifuged at $3000 \mathrm{rpm}$ for $5 \mathrm{~min}$ at $4{ }^{\circ} \mathrm{C}$. The absorbance of the supernatant was recorded at $650 \mathrm{~nm}$ using an ELx800 absorbance microplate reader (BioTek Instruments, Winooski, VT, USA). The inhibition activity was calculated as follows: inhibition $(\%)=100 \times[1-(\mathrm{As}-\mathrm{Abs}) /(\mathrm{Ac}-\mathrm{Acb})]$. Where: As is the absorbance of the sample (extract with enzyme), Asb is the absorbance of the sample blank (extract without enzyme), Ac is the absorbance of the control ( $100 \%$ enzyme activity, only solvent with enzyme), and Acb is the absorbance of the control blank ( $0 \%$ enzyme activity, only solvent without enzyme). Acarbose was used as a positive control.

$\alpha$-Glucosidase (G0660, Sigma-Aldrich, St. Louis, MO, USA) enzyme inhibitory effect of the extracts was determined according to the described method by Dat et al. (2021). In brief, the reaction mixture consisting of $50 \mu \mathrm{L}$ of the extract was incubated with $100 \mu \mathrm{L}$ of $0.1 \mathrm{M}$ potassium phosphate buffer $(\mathrm{pH} 6.8)$ containing $\alpha$-glucosidase solution $(0.5 \mathrm{U} / \mathrm{mL})$ in 96-well plates at $37^{\circ} \mathrm{C}$ for $10 \mathrm{~min}$. The reaction was started by adding $50 \mu \mathrm{L}$ of $5 \mathrm{mM} 4$ Nitrophenyl $\beta$-D-glucopyranoside (pNPG), followed by incubation at $37^{\circ} \mathrm{C}$ for $30 \mathrm{~min}$. The absorbance of released p-nitrophenol was recorded at $405 \mathrm{~nm}$ using an ELx800 absorbance microplate reader (BioTek Instruments, Winooski, VT, USA). The inhibition activity was calculated as follows: inhibition $(\%)=100 \times[1-(\mathrm{As}-\mathrm{Asb}) /(\mathrm{Ac}-\mathrm{Acb})]$. Where: As is the absorbance of the sample (extract with enzyme), and Asb is the absorbance of the sample blank (extract without enzyme), Ac is the absorbance of the control $(100 \%$ enzyme activity, only solvent with enzyme), and Acb is the absorbance of control blank ( $0 \%$ enzyme activity, only solvent without enzyme). Acarbose was used as a positive control.

\subsubsection{Xanthine Oxidase Inhibitory Activity}

The xanthine oxidase $(\mathrm{XO})$ inhibitory effect of the extracts was determined according to the method described by Nguyen et al. [87] with minor modifications. In brief, the reaction mixture consisting of $50 \mu \mathrm{L}$ of the extract, $35 \mu \mathrm{L}$ of $70 \mathrm{mM}$ phosphate buffer (pH7.5), and $30 \mu \mathrm{L}$ of enzyme solution $(0.01$ units $/ \mathrm{mL}$ in $70 \mathrm{mM}$ phosphate buffer, $\mathrm{pH} 7.5)$ was prepared immediately before use. Subsequently, the reaction mixture was preincubated at $25^{\circ} \mathrm{C}$ for $15 \mathrm{~min}$, and the reaction was then initiated by adding $60 \mu \mathrm{L}$ of substrate solution $(150 \mathrm{mM}$ xanthine in $70 \mathrm{mM}$ phosphate buffer, $\mathrm{pH} 7.5)$. The reaction mixture was incubated at $25^{\circ} \mathrm{C}$ for $30 \mathrm{~min}$, and the reaction was then stopped by adding $25 \mu \mathrm{L}$ of $1 \mathrm{~N} \mathrm{HCl}$. The absorbance of the mixture was measured at $290 \mathrm{~nm}$ using an ELx800 absorbance microplate reader (BioTek Instruments, Winooski, VT, USA). The XO inhibition activity was calculated as follows: inhibition $(\%)=100 \times[1-(\mathrm{As}-\mathrm{Asb}) /(\mathrm{Ac}-\mathrm{Acb})]$. Where: As is the absorbance of the sample (extract with enzyme), Asb is the absorbance of the sample blank (extract without enzyme), Ac is the absorbance of the control ( $100 \%$ enzyme activity, only solvent with enzyme), and Acb is the absorbance of the control blank ( $0 \%$ enzyme activity, only solvent without enzyme). Allopurinol was used as a positive control.

\subsubsection{Cytotoxic Activity}

Cytotoxic activity of the extracts against three cancer cell lines A549 (human lung carcinoma), MCF-7 (human breast carcinoma), and HeLa (human cervix carcinoma) was determined by Sulforhodamine B (SRB) assay as previously described by Skehan et al. [88]. Camptothecin was used as a positive control. 


\subsection{Gas Chromatography-Mass Spectrometry (GC-MS) Analysis}

GC-MS analyses were conducted using the Agilent 7890B gas chromatograph-assisted Agilent 5977A mass detector (Agilent Technologies, Stanta Clara, CA, USA). An HP-5MS capillary column $(30 \mathrm{~m} \times 0.250 \mathrm{~mm} \times 0.25 \mu \mathrm{M}$ film thickness; Agilent Technologies, Stanta Clara, CA, USA) was used for separation. The samples were diluted in hexane (1:10) and volumes of $1 \mu \mathrm{L}$ were injected (splitless mode) into the GC system at a flow rate of $1 \mathrm{~mL} / \mathrm{min}$. Helium $(99.999 \%$ of purity) was the carrier gas with a flow rate of $1 \mathrm{~mL} / \mathrm{min}$. The oven temperature was set at $60^{\circ} \mathrm{C}$ and held for $2 \mathrm{~min}$, then increased to $260^{\circ} \mathrm{C}$ at a rate of $5{ }^{\circ} \mathrm{C} / \mathrm{min}$ and held at this temperature for $1 \mathrm{~min}$. The inlet temperature was $260^{\circ} \mathrm{C}$ and the ionization source temperature was $280^{\circ} \mathrm{C}$. The solvent delay was $3.00 \mathrm{~min}$. The MS detector was operated in the EI mode at $70 \mathrm{eV}$, in the range of $m / z 50-550$, full scan mode. Data handling was performed using the Agilent ChemStation software C.01.10 (Agilent Technologies, Stanta Clara, CA, USA). The compounds were identified by comparing the spectra with a stored MS library (W8N08 and NIST08) with minimum matching quality of $90 \%$. The relative percent of individual components was calculated based on GC peak areas.

\subsection{Genome Sequencing, Assembly and Annotation of Biosynthesis Gene Clusters of Secondary Metabolites}

The endophytic bacterial strains were cultured in nutrient broth (NB, Himedia, Mumbai, India) overnight at $37^{\circ} \mathrm{C}$ under the shaking condition at $150 \mathrm{rpm}$. Cells were harvested by centrifugation at $10,000 \times g$ for $10 \mathrm{~min}$, and genomic DNA was isolated using the QIAamp DNA Mini Kit according to the manufacturer's protocol (QIAGEN, Hilden, Germany). The genome of the bacterial strains was sequenced by PacBio Sequel technology (PacBio, Menlo Park, CA, USA) according to the manufacturer's instructions.

The PacBio reads were assembled using HGAP4 (SMRT Link v.6.0.0.47841) [89]. The completeness of the genome assembly was estimated by Quality Assessment Tool for Genome Assemblies (QUAST v4.6.3; http:/ / bioinf.spbau.ru/quast/; accessed on 5 September 2021) [90] and Benchmarking Universal Single-Copy Orthologs (BUSCO v5.2.2, https://busco.ezlab.org/; accessed on 5 September 2021) [91]. Gene prediction for the complete genome sequence was performed using Prokka (v1.13) [92]. Genes involved in secondary metabolic pathways were predicted using secondary metabolite analysis shell (antiSMASH bacterial version v5.1.2, http:/ / antismash.secondarymetabolites.org/; accessed on 10 September 2021) [93]. The predicted biosynthetic gene clusters were also searched for previously known biosynthetic pathways using the Natural Product Domain Seeker (NaPdoS) (http:/ / napdos.ucsd.edu/; accessed on 10 September 2021) [94]. Biosynthetic gene cluster similarity networks and gene cluster families were generated using BiG-SCAPE (https: / /bigscape-corason.secondarymetabolites.org/; accessed on 10 September 2021) with default settings [95].

\section{Conclusions}

In the present study, twenty-three endophytic bacteria belonging to nine genera Streptomyces, Bacillus, Pseudovibrio, Microbacterium, Brevibacterium, Microbulbifer, Micrococcus, Rossellomorea, and Paracoccus were isolated from the fresh roots of the mangrove plant $R$. apiculata. The ethyl acetate extracts of the endophytic bacteria were evaluated for their pharmacological properties including antimicrobial, antioxidant, $\alpha$-amylase and $\alpha$-glucosidase inhibitory, xanthine oxidase inhibitory and cytotoxic activities. GC-MS analyses identified major volatile organic compounds from the ethyl acetate extract of the most potential strains Bacillus sp. RAR_GA_16, Rossellomorea vietnamensis RAR_WA_32, and Bacillus sp. RAR_M1_44. Genome studies found gene clusters related to the biosynthesis of secondary metabolites from the bacteria endophytes. Further investigations of the high bioactive strains-such as fermentation and isolation of pure bioactive compounds, and heterologous expression of novel BGCs in appropriate expression hosts-may allow exploring and exploiting the promising bioactive compounds for future drug development. 
Supplementary Materials: The following are available online at https:/ / www.mdpi.com/article/10 .3390/antibiotics10121491/s1, Table S1. Comparison of the GC-MS peaks of the extract of Bacillus sp. RAR_GA_16 with the spectra library. Table S2. Comparison of the GC-MS peaks of the extract of $R$. vietnamensis RAR_WA_32 with the spectra library. Table S3. Comparison of the GC-MS peaks of the extract of Bacillus sp. RAR_M1_44 with the spectra library.

Author Contributions: Conceptualization, T.T.H.D., H.L.T.A., P.V.C. conceived and designed the experiments; L.T.A. collected and identified the plant sample; T.T.H.D., L.C.V.C., and P.T.T.O. performed the experiments; T.T.H.D., L.C.V.C., L.T.H.M. contributed by analyzing data; T.T.H.D., H.H., L.T.L. analyzed genome data; T.T.H.D. prepared the draft manuscript. All authors have read and agreed to the published version of the manuscript.

Funding: This research was funded by the Vietnam Academy of Science and Technology, grant number ĐL0000.02/19-20.

Data Availability Statement: The 16S rRNA gene sequences of isolates are available in the NCBI database under accession numbers: MT229085-MT229104, OK649238-OK649240. The whole genome shotgun projects have been deposited at DDBJ/ENA/GenBank under the accession numbers: JAIUKX000000000JAIUKZ000000000. The versions described in this paper are versions JAIUKX010000000-JAIUKZ010000000.

Conflicts of Interest: The authors declare no conflict of interest.

\section{References}

1. Afzal, I.; Shinwari, Z.K.; Sikandar, S.; Shahzad, S. Plant beneficial endophytic bacteria: Mechanisms, diversity, host range and genetic determinants. Microbiol. Res. 2019, 221, 36-49. [CrossRef] [PubMed]

2. Cipollini, D.; Rigsby, C.M.; Barto, E.K. Microbes as Targets and Mediators of Allelopathy in Plants. J. Chem. Ecol. 2012, 38 , 714-727. [CrossRef] [PubMed]

3. Chuansheng, M.; Barry, S.F. The Use of Beneficial Microbial Endophytes for Plant Biomass and Stress Tolerance Improvement. Recent Pat. Biotechnol. 2010, 4, 81-95.

4. Rosenblueth, M.; Martínez-Romero, E. Bacterial Endophytes and Their Interactions with Hosts. Mol. Plant-Microbe Interact. 2006, 19, 827-837. [CrossRef]

5. Senthilkumar, M.; Anandham, R.; Madhaiyan, M.; Venkateswaran, V.; Sa, T. Endophytic Bacteria: Perspectives and Applications in Agricultural Crop Production. In Bacteria in Agrobiology: Crop Ecosystems; Maheshwari, D.K., Ed.; Springer: Berlin/Heidelberg, Germany, 2011; pp. 61-96.

6. Kathiresan, K.; Bingham, B.L. Biology of mangroves and mangrove Ecosystems. In Advances in Marine Biology; Academic Press: Cambridge, MA, USA, 2001; Volume 40, pp. 81-251.

7. Thatoi, H.; Behera, B.C.; Mishra, R.R.; Dutta, S.K. Biodiversity and biotechnological potential of microorganisms from mangrove ecosystems: A review. Ann. Microbiol. 2013, 63, 1-19. [CrossRef]

8. Cadamuro, R.D.; da Silveira Bastos, I.M.A.; Silva, I.T.; da Cruz, A.C.C.; Robl, D.; Sandjo, L.P.; Alves, S., Jr.; Lorenzo, J.M.; Rodríguez-Lázaro, D.; Treichel, H.; et al. Bioactive Compounds from Mangrove Endophytic Fungus and Their Uses for Microorganism Control. J. Fungi 2021, 7, 455. [CrossRef]

9. Kui-Wu, W.; Shi-Wei, W.; Bin, W.; Ji-Guang, W. Bioactive Natural Compounds from the Mangrove Endophytic Fungi. Mini-Rev. Med. Chem. 2014, 14, 370-391.

10. Ancheeva, E.; Daletos, G.; Proksch, P. Lead Compounds from Mangrove-Associated Microorganisms. Mar. Drugs 2018, 16, 370-391. [CrossRef]

11. Xu, D.-B.; Ye, W.-W.; Han, Y.; Deng, Z.-X.; Hong, K. Natural products from mangrove actinomycetes. Mar. Drugs 2014, 12, 2590-2613. [CrossRef]

12. Takayama, K.; Tateishi, Y.; Kajita, T. Global phylogeography of a pantropical mangrove genus Rhizophora. Sci. Rep. 2021, 11, 7228. [CrossRef]

13. Gao, M.-Z.; Yuan, X.-Y.; Cheng, M.-C.; Xiao, H.-B.; Bao, S.-X. A new diterpenoid from Rhizophora apiculata. J. Asian Nat. Prod. Res. 2011, 13, 776-779. [CrossRef]

14. Selvaraj, G.; Kaliamurthi, S.; Thirugnasambandan, R. Effect of Glycosin alkaloid from Rhizophora apiculata in non-insulin dependent diabetic rats and its mechanism of action: In vivo and in silico studies. Phytomedicine 2016, 23, 632-640. [CrossRef]

15. Moovendhan, M.; Ramasubburayan, R.; Vairamani, S.; Shanmugam, A.; Palavesam, A.; Immanuel, G. Antibiotic susceptibility of Genistein and Alkaloids from Rhizophora apiculata. Biocatal. Agric. Biotechnol. 2014, 3, 323-327. [CrossRef]

16. Saxena, E.; Garg, H.S. Apiculol, 1ß-hydroxy-13-epimanoyl Oxide from Roots of Rhizophora apiculata Mangrove. Nat. Prod. Lett. 1994, 4, 149-154. [CrossRef]

17. Kokpol, U.; Chavasiri, W.; Chittawong, V.; Bruce, M.; Cunningham, G.N.; Miles, D.H. Long chain aliphatic alcohols and saturated carboxylic acids from heartwood of Rhizophora apiculata. Phytochemistry 1993, 33, 1129-1131. [CrossRef]

18. Ramalingam, V.; Rajaram, R. Enhanced antimicrobial, antioxidant and anticancer activity of Rhizophora apiculata: An experimental report. 3 Biotech 2018, 8, 200. [CrossRef] [PubMed] 
19. Loo, A.Y.; Jain, K.; Darah, I. Antioxidant activity of compounds isolated from the pyroligneous acid, Rhizophora apiculata. Food Chem. 2008, 107, 1151-1160. [CrossRef]

20. Gao, M.; Xiao, H. Activity-guided isolation of antioxidant compounds from Rhizophora apiculata. Molecules 2012, 17, 10675-10682. [CrossRef]

21. Vijayavel, K.; Anbuselvam, C.; Balasubramanian, M.P. Free radical scavenging activity of the marine mangrove Rhizophora apiculata bark extract with reference to naphthalene induced mitochondrial dysfunction. Chem. Biol. Interact. 2006, 163, 170-175. [CrossRef]

22. Premanathan, M.; Arakaki, R.; Izumi, H.; Kathiresan, K.; Nakano, M.; Yamamoto, N.; Nakashima, H. Antiviral properties of a mangrove plant, Rhizophora apiculata Blume, against human immunodeficiency virus. Antivir. Res. 1999, 44, 113-122. [CrossRef]

23. Zhang, H.; Jacob, J.A.; Jiang, Z.; Xu, S.; Sun, K.; Zhong, Z.; Varadharaju, N.; Shanmugam, A. Hepatoprotective effect of silver nanoparticles synthesized using aqueous leaf extract of Rhizophora apiculata. Int. J. Nanomed. 2019, 14, 3517-3524. [CrossRef]

24. Fan, Y.; Wang, Y.; Liu, P.; Fu, P.; Zhu, T.; Wang, W.; Zhu, W. Indole-Diterpenoids with Anti-H1N1 Activity from the Aciduric Fungus Penicillium camemberti OUCMDZ-1492. J. Nat. Prod. 2013, 76, 1328-1336. [CrossRef] [PubMed]

25. Chaeprasert, S.; Piapukiew, J.; Whalley, A.J.S.; Sihanonth, P. Endophytic fungi from mangrove plant species of Thailand: Their antimicrobial and anticancer potentials. Bot. Mar. 2010, 53, 555-564. [CrossRef]

26. Zhou, G.; Qiao, L.; Zhang, X.; Sun, C.; Che, Q.; Zhang, G.; Zhu, T.; Gu, Q.; Li, D. Fusaricates H-K and fusolanones A-B from a mangrove endophytic fungus Fusarium solani HDN15-410. Phytochemistry 2019, 158, 13-19. [CrossRef]

27. UNEP. National Reports on Mangroves in the South China Sea; UNEP/GEF/SCS Technical Publication No. 14; UNEP: Nairobi, Kenya, 2008.

28. Putra, M.Y.; Karim, F. Antibacterial and antioxidant activity-guided isolation studies on Fusarium sp. associated with the ascidian Botryllus schlosseri. AIP Conf. Proc. 2020, 2243, 020019.

29. Ser, H.-L.; Palanisamy, U.D.; Yin, W.-F.; Abd Malek, S.N.; Chan, K.-G.; Goh, B.-H.; Lee, L.-H. Presence of antioxidative agent, Pyrrolo[1,2-a]pyrazine-1,4-dione, hexahydro- in newly isolated Streptomyces mangrovisoli sp. nov. Front. Microbiol. 2015, 6, 854. [CrossRef] [PubMed]

30. Zhai, Y.; Shao, Z.; Cai, M.; Zheng, L.; Li, G.; Yu, Z.; Zhang, J. Cyclo(1-Pro-1-Leu) of Pseudomonas putida MCCC 1 A00316 Isolated from Antarctic Soil: Identification and Characterization of Activity against Meloidogyne incognita. Molecules 2019, $24,768$. [CrossRef]

31. Rhee, K.-H. Cyclic dipeptides exhibit synergistic, broad spectrum antimicrobial effects and have anti-mutagenic properties. Int. J. Antimicrob. Agents 2004, 24, 423-427. [CrossRef] [PubMed]

32. Nishanth Kumar, S.; Mohandas, C.; Siji, J.V.; Rajasekharan, K.N.; Nambisan, B. Identification of antimicrobial compound, diketopiperazines, from a Bacillus sp. $\mathrm{N}$ strain associated with a rhabditid entomopathogenic nematode against major plant pathogenic fungi. J. Appl. Microbiol. 2012, 113, 914-924. [CrossRef]

33. Casillas-Vargas, G.; Ocasio-Malavé, C.; Medina, S.; Morales-Guzmán, C.; Del Valle, R.G.; Carballeira, N.M.; Sanabria-Ríos, D.J. Antibacterial fatty acids: An update of possible mechanisms of action and implications in the development of the next-generation of antibacterial agents. Prog. Lipid Res. 2021, 82, 101093. [CrossRef]

34. Chen, S.; Liu, J.; Gong, H.; Yang, D. Identification and antibacterial activity of secondary metabolites from Taxus endophytic fungus. Sheng Wu Gong Cheng Xue Bao 2009, 25, 368-374.

35. Rahman, S.; Anwar, M.N. Fungitoxic and cytotoxic activity of a novel compound 1,2-benzenedicarboxylic acid, diiooctyl ester of Plumbago zeylanica Linn. Asian J. Microbiol. Biotechnol. Environ. Sci. 2006, 8, 461-464.

36. Zheng, C.J.; Yoo, J.-S.; Lee, T.-G.; Cho, H.-Y.; Kim, Y.-H.; Kim, W.-G. Fatty acid synthesis is a target for antibacterial activity of unsaturated fatty acids. FEBS Lett. 2005, 579, 5157-5162. [CrossRef]

37. Galbraith, H.; Miller, T.B.; Paton, A.M.; Thompson, J.K. Antibacterial Activity of Long Chain Fatty Acids and the Reversal with Calcium, Magnesium, Ergocalciferol and Cholesterol. J. Appl. Bacteriol. 1971, 34, 803-813. [CrossRef] [PubMed]

38. Almoughrabie, S.; Ngari, C.; Guillier, L.; Briandet, R.; Poulet, V.; Dubois-Brissonnet, F. Rapid assessment and prediction of the efficiency of two preservatives against $S$. aureus in cosmetic products using High Content Screening-Confocal Laser Scanning Microscopy. PLoS ONE 2020, 15, e0236059. [CrossRef]

39. Lucchini, J.J.; Corre, J.; Cremieux, A. Antibacterial activity of phenolic compounds and aromatic alcohols. Res. Microbiol. 1990, 141, 499-510.

40. Zou, J.; Gao, P.; Hao, X.; Xu, H.; Zhan, P.; Liu, X. Recent progress in the structural modification and pharmacological activities of ligustrazine derivatives. Eur. J. Med. Chem. 2018, 147, 150-162. [CrossRef]

41. Yan, S.; Yue, Y.-Z.; Zong, Y.; Zeng, L. Tetramethylpyrazine Improves Postoperative Tissue Adhesion: A Drug Repurposing. Chin. J. Integr. Med. 2019, 25, 554-560. [CrossRef] [PubMed]

42. Yang, W.-t.; Zheng, X.-w.; Chen, S.; Shan, C.-S.; Xu, Q.-Q.; Zhu, J.-Z.; Bao, X.-Y.; Lin, Y.; Zheng, G.-q.; Wang, Y. Chinese herbal medicine for Alzheimer's disease: Clinical evidence and possible mechanism of neurogenesis. Biochem. Pharmacol. 2017, 141, 143-155. [CrossRef] [PubMed]

43. Corre, J.; Lucchini, J.J.; Mercier, G.M.; Cremieux, A. Antibacterial activity of phenethyl alcohol and resulting membrane alterations. Res. Microbiol. 1990, 141, 483-497. [CrossRef]

44. Fraud, S.; Rees, E.L.; Mahenthiralingam, E.; Russell, A.D.; Maillard, J.Y. Aromatic alcohols and their effect on Gram-negative bacteria, cocci and mycobacteria. J. Antimicrob. Chemother. 2003, 51, 1435-1436. [CrossRef] [PubMed] 
45. Davidson, P.M.; Sofos, J.N.; Branen, A.L. Antimicrobials in Food, 3rd ed.; CRC Press: Boca Raton, FL, USA, 2005.

46. Wu, J.-J.; Huang, J.-W.; Deng, W.-L. Phenylacetic Acid and Methylphenyl Acetate From the Biocontrol Bacterium Bacillus mycoides BM02 Suppress Spore Germination in Fusarium oxysporum f. sp. lycopersici. Front. Microbiol. 2020, 11, 3022. [CrossRef]

47. Kleinwächter, I.S.; Pannwitt, S.; Centi, A.; Hellmann, N.; Thines, E.; Bereau, T.; Schneider, D. The Bacteriostatic Activity of 2-Phenylethanol Derivatives Correlates with Membrane Binding Affinity. Membranes 2021, 11, 254. [CrossRef] [PubMed]

48. Hwang Byung, K.; Lim Song, W.; Kim Beom, S.; Lee Jung, Y.; Moon Surk, S. Isolation and In Vivo and In Vitro Antifungal Activity of Phenylacetic Acid and Sodium Phenylacetate from Streptomyces humidus. Appl. Environ. Microbiol. 2001, 67, 3739-3745. [CrossRef]

49. Kim, Y.; Cho, J.-Y.; Kuk, J.-H.; Moon, J.-H.; Cho, J.-I.; Kim, Y.-C.; Park, K.-H. Identification and Antimicrobial Activity of Phenylacetic Acid Produced by Bacillus licheniformis Isolated from Fermented Soybean, Chungkook-Jang. Curr. Microbiol. 2004, 48, 312-317. [CrossRef]

50. Narayana, K.J.P.; Prabhakar, P.; Vijayalakshmi, M.; Venkateswarlu, Y.; Krishna, P.S.J. Biological activity of phenylpropionic acid isolated from a terrestrial Streptomycetes. Pol. J. Microbiol. 2007, 56, 191-197.

51. Varsha, K.K.; Devendra, L.; Shilpa, G.; Priya, S.; Pandey, A.; Nampoothiri, K.M. 2,4-Di-tert-butyl phenol as the antifungal, antioxidant bioactive purified from a newly isolated Lactococcus sp. Int. J. Food Microbiol. 2015, 211, 44-50. [CrossRef] [PubMed]

52. Dharni, S.; Maurya, A.; Samad, A.; Srivastava, S.K.; Sharma, A.; Patra, D.D. Purification, Characterization, and in Vitro Activity of 2,4-Di-tert-butylphenol from Pseudomonas monteilii PsF84: Conformational and Molecular Docking Studies. J. Agric. Food Chem. 2014, 62, 6138-6146. [CrossRef] [PubMed]

53. Chen, X.; Zhao, X.; Deng, Y.; Bu, X.; Ye, H.; Guo, N. Antimicrobial potential of myristic acid against Listeria monocytogenes in milk. J. Antibiot. 2019, 72, 298-305. [CrossRef]

54. Huang, C.B.; Alimova, Y.; Myers, T.M.; Ebersole, J.L. Short- and medium-chain fatty acids exhibit antimicrobial activity for oral microorganisms. Arch. Oral Biol. 2011, 56, 650-654. [CrossRef]

55. Jeon, Y.-T.; Jun, E.-M.; Oh, K.-B.; Thu, P.Q.; Kim, S.-U. Identification of 12-methyltetradecanoic acid from endophytic Senotrophomonas maltophilia as inhibitor of appressorium formation of Magnaporthe oryzae. J. Korean Soc. Appl. Biol. Chem. 2010, 53, 578-583. [CrossRef]

56. Yang, P.; Collin, P.; Madden, T.; Chan, D.; Sweeney-Gotsch, B.; McConkey, D.; Newman, R.A. Inhibition of proliferation of PC3 cells by the branched-chain fatty acid, 12-methyltetradecanoic acid, is associated with inhibition of 5-lipoxygenase. Prostate 2003, 55, 281-291. [CrossRef]

57. Saeed, N.M.; El-Demerdash, E.; Abdel-Rahman, H.M.; Algandaby, M.M.; Al-Abbasi, F.A.; Abdel-Naim, A.B. Anti-inflammatory activity of methyl palmitate and ethyl palmitate in different experimental rat models. Toxicol. Appl. Pharmacol. 2012, 264, 84-93. [CrossRef]

58. Shaaban, M.T.; Ghaly, M.F.; Fahmi, S.M. Antibacterial activities of hexadecanoic acid methyl ester and green-synthesized silver nanoparticles against multidrug-resistant bacteria. J. Basic Microbiol. 2021, 61, 557-568. [CrossRef] [PubMed]

59. Dilika, F.; Bremner, P.D.; Meyer, J.J.M. Antibacterial activity of linoleic and oleic acids isolated from Helichrysum pedunculatum: A plant used during circumcision rites. Fitoterapia 2000, 71, 450-452. [CrossRef]

60. Ström, K.; Sjögren, J.; Broberg, A.; Schnürer, J. Lactobacillus plantarum MiLAB 393 Produces the Antifungal Cyclic Dipeptides Cyclo(1-Phe-1-Pro) and Cyclo(1-Phe-trans-4-OH-1-Pro) and 3-Phenyllactic Acid. Appl. Environ. Microbiol. 2002, 68, 4322-4327. [CrossRef]

61. Qi, S.-H.; Xu, Y.; Gao, J.; Qian, P.-Y.; Zhang, S. Antibacterial and antilarval compounds from marine bacterium Pseudomonas rhizosphaerae. Ann. Microbiol. 2009, 59, 229. [CrossRef]

62. Brauns, S.C.; Milne, P.; Naudé, R.; Van de Venter, M. Selected cyclic dipeptides inhibit cancer cell growth and induce apoptosis in HT-29 colon cancer cells. Anticancer Res. 2004, 24, 1713-1719.

63. Singh, V.K.; Mishra, A.; Jha, B. 3-Benzyl-Hexahydro-Pyrrolo[1,2-a]Pyrazine-1,4-Dione Extracted From Exiguobacterium indicum Showed Anti-biofilm Activity Against Pseudomonas aeruginosa by Attenuating Quorum Sensing. Front. Microbiol. $2019,10,1269$. [CrossRef] [PubMed]

64. Sastry, V.M.V.S.; Rao, G.R.K. Dioctyl phthalate, and antibacterial compound from the marine brown alga —Sargassum wightii. J. Appl. Phycol. 1995, 7, 185-186. [CrossRef]

65. Kim, S.-K.; Kim, S.-S.; Kim, K.-S.; Chung, Y.-R.; Kim, C.-H. New Antibiotics Produced by Streptomyces melanosporofaciens II. Antimicrobial Activities and Isolation, Purification, and Structure Determination of the Active Compound. Kor. J. Microbiol. Biotechnol. 1991, 19, 235-241.

66. Nguyen, D.T.M.; Nguyen, D.H.; Hwa-La, L.; Lee, H.; Shin, J.-H.; Kim, E.-I. Inhibition of Melanogenesis by Dioctyl Phthalate Isolated from Nigella glandulifera Freyn. J. Microbiol. Biotechnol. 2007, 17, 1585-1590.

67. U.S. Department of Agriculture Agricultural Research Service Dr. Duke's Phytochemical and Ethnobotanical Databases. Available online: http:/ / phytochem.nal.usda.gov/ (accessed on 25 October 2021).

68. Hamazaki, K.; Suzuki, N.; Kitamura, K.-i.; Hattori, A.; Nagasawa, T.; Itomura, M.; Hamazaki, T. Is vaccenic acid (18:1t n-7) associated with an increased incidence of hip fracture? An explanation for the calcium paradox. Prostaglandins Leukot. Essent. Fat. Acids 2016, 109, 8-12. [CrossRef]

69. Bibi, S.N.; Gokhan, Z.; Rajesh, J.; Mahomoodally, M.F. Fungal endophytes associated with mangroves-Chemistry and biopharmaceutical potential. S. Afr. J. Bot. 2020, 134, 187-212. [CrossRef] 
70. Dat, T.T.H.; Oanh, P.T.T. In vitro antioxidant, $\alpha$-amylase and $\alpha$-glucosidase inhibitory activities of endophytic bacteria from the roots of the mangrove plant Rhizophora stylosa Griffith. Acad. J. Biol. 2021, 43, 125-135. [CrossRef]

71. Sebastianes, F.L.S.; de Azevedo, J.L.; Lacava, P.T. Diversity and Biotechnological Potential of Endophytic Microorganisms Associated with Tropical Mangrove Forests. In Diversity and Benefits of Microorganisms from the Tropics; de Azevedo, J.L., Quecine, M.C., Eds.; Springer International Publishing: Cham, Switzerland, 2017; pp. 37-56.

72. Castro, R.A.; Dourado, M.N.; Almeida, J.R.D.; Lacava, P.T.; Nave, A.; Melo, I.S.d.; Azevedo, J.L.D.; Quecine, M.C. Mangrove endophyte promotes reforestation tree (Acacia polyphylla) growth. Braz. J. Microbiol. 2018, 49, 59-66. [CrossRef] [PubMed]

73. Dat, T.T.H.; Oanh, P.T.T.; Tam, V.T.T.; Anh, H.L.T. Antimicrobial and antioxidant activity of bacterial endophytes isolated from leaves of the mangrove plant Rhizophora stylosa. Acad. J. Biol. 2019, 41, 91-99. [CrossRef]

74. Hernández-González, I.L.; Moreno-Hagelsieb, G.; Olmedo-Álvarez, G. Environmentally-driven gene content convergence and the Bacillus phylogeny. BMC Evol. Biol. 2018, 18, 148. [CrossRef] [PubMed]

75. Ian, E.; Malko, D.B.; Sekurova, O.N.; Bredholt, H.; Rückert, C.; Borisova, M.E.; Albersmeier, A.; Kalinowski, J.; Gelfand, M.S.; Zotchev, S.B. Genomics of Sponge-Associated Streptomyces spp. Closely Related to Streptomyces albus J1074: Insights into Marine Adaptation and Secondary Metabolite Biosynthesis Potential. PLoS ONE 2014, 9, e96719.

76. Versluis, D.; Nijsse, B.; Naim, M.A.; Koehorst, J.J.; Wiese, J.; Imhoff, J.F.; Schaap, P.J.; van Passel, M.W.J.; Smidt, H.; Sipkema, D. Comparative Genomics Highlights Symbiotic Capacities and High Metabolic Flexibility of the Marine Genus Pseudovibrio. Genome Biol. Evol. 2018, 10, 125-142. [CrossRef]

77. Steil, L.; Hoffmann, T.; Budde, I.; Völker, U.; Bremer, E. Genome-Wide Transcriptional Profiling Analysis of Adaptation of Bacillus subtilis to High Salinity. J. Bacteriol. 2003, 185, 6358. [CrossRef]

78. Tian, X.; Zhang, Z.; Yang, T.; Chen, M.; Li, J.; Chen, F.; Yang, J.; Li, W.; Zhang, B.; Zhang, Z.; et al. Comparative Genomics Analysis of Streptomyces Species Reveals Their Adaptation to the Marine Environment and Their Diversity at the Genomic Level. Front. Microbiol. 2016, 7, 998. [CrossRef]

79. Bondarev, V.; Richter, M.; Romano, S.; Piel, J.; Schwedt, A.; Schulz-Vogt, H.N. The genus Pseudovibrio contains metabolically versatile bacteria adapted for symbiosis. Environ. Microbiol. 2013, 15, 2095-2113. [CrossRef] [PubMed]

80. Belbahri, L.; Chenari Bouket, A.; Rekik, I.; Alenezi, F.N.; Vallat, A.; Luptakova, L.; Petrovova, E.; Oszako, T.; Cherrad, S.; Vacher, S.; et al. Comparative Genomics of Bacillus amyloliquefaciens Strains Reveals a Core Genome with Traits for Habitat Adaptation and a Secondary Metabolites Rich Accessory Genome. Front. Microbiol. 2017, 8, 1438. [CrossRef] [PubMed]

81. Rukachaisirikul, V.; Rodglin, A.; Sukpondma, Y.; Phongpaichit, S.; Buatong, J.; Sakayaroj, J. Phthalide and Isocoumarin Derivatives Produced by an Acremonium sp. Isolated from a Mangrove Rhizophora apiculata. J. Nat. Prod. 2012, 75, 853-858. [CrossRef]

82. Klaiklay, S.; Rukachaisirikul, V.; Tadpetch, K.; Sukpondma, Y.; Phongpaichit, S.; Buatong, J.; Sakayaroj, J. Chlorinated chromone and diphenyl ether derivatives from the mangrove-derived fungus Pestalotiopsis sp. PSU-MA69. Tetrahedron 2012, 68, $2299-2305$. [CrossRef]

83. Klaiklay, S.; Rukachaisirikul, V.; Phongpaichit, S.; Pakawatchai, C.; Saithong, S.; Buatong, J.; Preedanon, S.; Sakayaroj, J. Anthraquinone derivatives from the mangrove-derived fungus Phomopsis sp. PSU-MA214. Phytochem. Lett. 2012, 5, 738-742. [CrossRef]

84. Klaiklay, S.; Rukachaisirikul, V.; Phongpaichit, S.; Buatong, J.; Preedanon, S.; Sakayaroj, J. Flavodonfuran: A new difuranylmethane derivative from the mangrove endophytic fungus Flavodon flavus PSU-MA201. Nat. Prod. Res. 2013, 27, 1722-1726. [CrossRef] [PubMed]

85. Dat, T.T.H.; Cuc, N.T.; Cuong, P.V.; Smidt, H.; Sipkema, D. Diversity and Antimicrobial Activity of Vietnamese Sponge-Associated Bacteria. Mar. Drugs 2021, 19, 353. [CrossRef]

86. Lane, D.J. 16S/23S rRNA Sequencing. In Nucleic Acid Techniques in Bacterial Systematics; Stackebrandt, E., Goodfellow, M., Eds.; John Wiley \& Sons: New York, NY, USA, 1991; pp. 115-175.

87. Nguyen, M.T.T.; Awale, S.; Tezuka, Y.; Tran, Q.L.; Watanabe, H.; Kadota, S. Xanthine Oxidase Inhibitory Activity of Vietnamese Medicinal Plants. Biol. Pharm. Bull. 2004, 27, 1414-1421. [CrossRef]

88. Skehan, P.; Storeng, R.; Scudiero, D.; Monks, A.; McMahon, J.; Vistica, D.; Warren, J.T.; Bokesch, H.; Kenney, S.; Boyd, M.R. New Colorimetric Cytotoxicity Assay for Anticancer-Drug Screening. JNCI J. Natl. Cancer Inst. 1990, 82, 1107-1112. [CrossRef] [PubMed]

89. Chin, C.-S.; Peluso, P.; Sedlazeck, F.J.; Nattestad, M.; Concepcion, G.T.; Clum, A.; Dunn, C.; O’Malley, R.; Figueroa-Balderas, R.; Morales-Cruz, A.; et al. Phased diploid genome assembly with single-molecule real-time sequencing. Nat. Methods 2016, 13, 1050-1054. [CrossRef] [PubMed]

90. Mikheenko, A.; Valin, G.; Prjibelski, A.; Saveliev, V.; Gurevich, A. Icarus: Visualizer for de novo assembly evaluation. Bioinformatics 2016, 32, 3321-3323. [CrossRef] [PubMed]

91. Manni, M.; Berkeley, M.R.; Seppey, M.; Simão, F.A.; Zdobnov, E.M. BUSCO Update: Novel and Streamlined Workflows along with Broader and Deeper Phylogenetic Coverage for Scoring of Eukaryotic, Prokaryotic, and Viral Genomes. Mol. Biol. Evol. 2021, 38, 4647-4654. [CrossRef]

92. Seemann, T. Prokka: Rapid prokaryotic genome annotation. Bioinformatics 2014, 30, 2068-2069. [CrossRef]

93. Blin, K.; Shaw, S.; Steinke, K.; Villebro, R.; Ziemert, N.; Lee, S.Y.; Medema, M.H.; Weber, T. antiSMASH 5.0: Updates to the secondary metabolite genome mining pipeline. Nucleic Acids Res. 2019, 47, W81-W87. [CrossRef] 
94. Ziemert, N.; Podell, S.; Penn, K.; Badger, J.H.; Allen, E.; Jensen, P.R. The Natural Product Domain Seeker NaPDoS: A Phylogeny Based Bioinformatic Tool to Classify Secondary Metabolite Gene Diversity. PLoS ONE 2012, 7, e34064.

95. Navarro-Muñoz, J.C.; Selem-Mojica, N.; Mullowney, M.W.; Kautsar, S.A.; Tryon, J.H.; Parkinson, E.I.; De Los Santos, E.L.C.; Yeong, M.; Cruz-Morales, P.; Abubucker, S.; et al. A computational framework to explore large-scale biosynthetic diversity. Nat. Chem. Biol. 2020, 16, 60-68. [CrossRef] 